\title{
Rapid degradation and 3D CLEM of condensin reveal chromatin compaction uncoupled from chromosome architecture in mitosis
}

Kumiko Samejima ${ }^{1 * \ddagger}$, Daniel G. Booth ${ }^{1 x^{*}}$, Hiromi Ogawa ${ }^{1 \$}$, James R. Paulson ${ }^{2}$, Linfeng $\mathrm{Xie}^{2}$, Cara A. Watson ${ }^{1 \wedge}$, Melpomeni Platani ${ }^{1}$, Masato T. Kanemaki ${ }^{3}$, and William C. Earnshaw ${ }^{1 \ddagger}$

${ }^{1}$ Wellcome Centre for Cell Biology

University of Edinburgh, King's Buildings, Max Born Crescent

Edinburgh EH9 3BF, Scotland, UK

${ }^{2}$ Department of Chemistry, University of Wisconsin-Oshkosh, 800 Algoma Blvd, Oshkosh, WI 54901 USA.

${ }^{3}$ Division of Molecular Cell Engineering, National Institute of Genetics, ROIS, and Department of Genetics, SOKENDAI, Yata 1111, Mishima, Shizuoka 411-8540, Japan

Present address:

${ }^{x}$ Centre For Neuroregeneration, University of Edinburgh, The Chancellor's Building, 49 Little France Crescent, Edinburgh, EH16 4SB

\$Area of Green and Environmental Innovation, Division of Strategic Research and Development, Graduate School of Science and Engineering, Saitama University, 255, Shimo-ohkubo, Sakura-ku, Saitama-shi, Saitama, 338-8570, Japan

^Merial Animal Health Ltd., Sandringham House, Harlow Business Park, Harlow, CM19 5QE, England, UK

*These authors contributed equally to this study

‡Corresponding authors

bill.earnshaw@ed.ac.uk

kumiko.samejima@ed.ac.uk 


\section{Abstract:}

The requirement for condensin in chromosome formation in somatic cells remains unclear as imperfectly condensed chromosomes do form in conventional condensindepleted cells. Here we have dissected the role of condensin at different stages of vertebrate mitosis by combining auxin-mediated rapid depletion of condensin subunit SMC2 with chemical genetics to obtain near-synchronous mitotic entry of chicken DT40 cells. We analysed the outcomes by live and fixed-cell microscopy methods, including 3D correlative light and serial block face scanning electron microscopy. Following rapid depletion of condensin, chromosomal defects were obvious. The chromatin was compacted normally, but formed a single mass of mitotic chromosomes clustered at one side of a bent mitotic spindle. Cultures arrest at prometaphase, eventually exiting mitosis without segregating chromosomes. Experiments titrating the auxin concentration suggest a previously unsuspected dual role of condensin, as different condensin levels are required for anaphase chromosome segregation and formation of a normal chromosome architecture. 


\section{INTRODUCTION:}

As cells enter mitosis, the chromatin is compacted 2-3-fold in volume as it undergoes a dramatic re-organisation (Lleres et al., 2009; Martin and Cardoso, 2010;

4 Vagnarelli and Earnshaw, 2012). Rod-shaped mitotic chromosomes form and chromosome scaffold proteins are localised along the sister chromatid axes (Paulson and Laemmli, 1977; Lewis and Laemmli, 1982; Earnshaw et al., 1985; Gasser et al., 1986; Saitoh et al., 1994; Samejima et al., 2012). These proteins include topoisomerase Il $\alpha$, chromokinesin KIF4 and SMC2 (Structural Maintenance of Chromosomes 2), a core subunit of both condensin complexes (Earnshaw et al., 1985; Gasser and Laemmli, 1986; Saitoh et al., 1994; Hirano and Mitchison, 1994; Hirano et al., 1997; Ono et al., 2003; Samejima et al., 2012). lagging chromosomes blocking cytokinesis in all the experimental systems tested including yeasts, D. melanogaster, nematodes, and vertebrate cells (Saka et al., 1994; Steffensen et al., 2001; Hagstrom et al., 2002; Hudson et al., 2003) (Houlard et al., 2015). Mitotic chromosome structural defects are also commonly observed. However, the extent of those defects varies between different experimental systems (Hirano and Mitchison, 1994; Hirano et al., 1997; Saka et al., 1994; Bhat et al., 1996; Steffensen et al., 2001; Hagstrom et al., 2002; Somma et al., 2003; Hudson et al., 2003; Coelho et al., 2003; Vagnarelli et al., 2006; Maddox et al., 2006; Samoshkin et al., 2009). At one extreme, immuno-depletion of condensins from Xenopus egg extracts results in the formation of a decondensed chromosome mass, indicating that condensins are essential for assembly and maintenance of mitotic chromosome structure (Hirano and Mitchison, 1994; Hirano et al., 1997). S. pombe temperaturesensitive mutants of condensin subunits also fail to condense chromosomes under restrictive conditions (Saka et al., 1994; Sutani et al., 1999). Meiotic I oocytes depleted of condensin subunits showed massively deformed chromosomes (Houlard 2015).

At the other extreme, vertebrate cells depleted of condensins using conditional knockouts or siRNA exhibit relatively moderate defects (Hudson et al.,

31 2003; Ono et al., 2003; Ono et al., 2004; Vagnarelli et al., 2006; Samoshkin et al., 
33 structural rigidity seen in wild type chromosomes. These inconsistent phenotypes

34 among different experimental systems pose a "condensin paradox" (Gassmann et al.,

35 2004), suggesting that condensin might not be universally required for mitotic

36 chromosome formation.

In the Xenopus egg extract experiments, condensin was immuno-depleted before the addition of sperm DNA. In the case of S. pombe, condensin was rapidly inactivated by a temperature shift. In contrast, vertebrate condensin was gradually lost by natural turnover over more than one cell cycle after synthesis of new protein was halted. Thus, the milder defects in chromosome assembly seen in vertebrates might be explained by cellular adaptation to the gradual loss and/or incomplete depletion of condensin [see e.g. (Wood et al., 2016)]. Furthermore, the various mitotic defects observed could result from a mixture of primary and secondary effects or even non-mitotic functions of condensin (Hirano, 2016). Together, these observations suggested that rapid depletion of condensin in vertebrate cells might more accurately reveal its true mitotic function(s).

This rapid depletion is now possible using an Auxin Inducible Degron (AID) system (Nishimura et al., 2009; Kanemaki, 2013). The plant hormone auxin enhances the affinity of the plant-specific F-box protein OsTIR1 for the AID tag AtIAA17. In the presence of auxin, tagged target proteins become poly-ubiquitylated and are degraded rapidly via the ubiquitin-proteasome pathway. It can take as little as $1 \mathrm{~h}$ to deplete a target protein in vertebrate cells. Furthermore, the AID system allowed us to study cells partially depleted of condensin by titrating the amount of auxin (Nishimura 2009). This is difficult or impossible using the TEV protease cleavage mediated condensin inactivation system. chromosome morphology changes on a minute-by-minute basis as cells enter mitosis. However, prophase cells comprise less than $1 \%$ of the population in asynchronous cultures. Thus, cell synchrony protocols are needed. Importantly,

61 commonly used nocodazole or colcemid treatments are not suitable for studying

62 chromosome morphology, as both treatments delay cells in prometaphase and 63 induce hyper-condensation of the chromosomes. 

An alternative strategy is to synchronise cultures before they enter mitosis.

65 Regulated CDK1 activity is essential for mitotic entry and progression. Manipulation

66 of this activity can be used to enrich for mitotic cells (Nurse and Bissett, 1981);

67 (Vassilev et al., 2006; Smith et al., 2011). To this end, an analogue sensitive cyclin

68 dependent kinase $1\left(\mathrm{CDK}^{\mathrm{as}}\right)$ system was established to specifically inhibit CDK1

69 activity (Hochegger et al., 2007). CDK1 ${ }^{\text {as }}$ has a mutation in the ATP binding pocket

70 allowing the binding of 1NMPP1, an ATP analogue with a bulky side chain (Bishop et

71 al., 2000). Addition of 1 NMPP1 blocks $C D K 1^{\text {as }}$ cells in late $G 2$, and upon washout of

72 the drug, the cells swiftly enter mitosis.

73 Here we have used rapid degradation of SMC2 in highly synchronized

74 cultures coupled with 3D-CLEM (correlative light and electron microscopy) to probe

75 the function of condensin in mitotic chromosome structure and segregation. We find

76 that mitotic chromatin is compacted to the normal extent though the intrinsic

77 chromosome architecture is faulty in condensin-depleted cells. We also find that

78 differing levels of condensin are required for normal chromosome architecture,

79 sister chromatid axis formation and chromosome segregation at anaphase.

80 
81

82

83

84

85

86

87

88

89

90

91

92

93

94

95

96

97

98

99

100

101

102

103

104

105

106

107

108

109

110

111

112

RESULTS:

\section{SMC2-AID-GFP cell line to study the mitotic roles of condensin}

Defining the roles of condensin in vertebrate cells during mitosis has been difficult due to relatively mild defects observed following depletion of condensin subunits in mitotic cells of numerous species, compared to defects seen in vitro, in yeasts, in meiotic mouse oocytes and in fly embryos. This could be explained by variable levels of residual condensin in different experimental systems or by the cells adapting to the gradual loss of condensin. We hypothesized that rapid depletion of a key condensin subunit might resolve this issue. In order to test this hypothesis, we decided to modify SMC2 (condensin I and II common subunit) to be rapidly depleted in chicken DT40 cells. This is because both condensin I and II have important roles in mitotic chromosome assembly in chicken DT40 cells similar to human cultured cells (Ono 2003, Green 2012) and also we could directly compare the defects with those of our chicken DT40 conditional SMC2 knockouts (SMC2 ${ }^{\text {ON/OFF }) ~(H u d s o n ~ 2003) . ~}$

Taking advantage of an inducible degron (AID) system, we established the SMC2-AID-GFP cell line expressing a SMC2 CDNA fused with a minimal AID degron and GFP tag as the sole source of SMC2 together with the auxin-responsive F-box protein TIR1 (Figure 1A and see Materials and Methods). SMC2-AID-GFP cells proliferated well only in the absence of auxin (Figure 1B). In contrast, addition of auxin did not affect the growth rate of wild type cells. Mitotic index (MI), mitotic profile and cell cycle profile of the SMC2-AID-GFP cells were similar to the ones of wild type cells without auxin (Figure 1-figure supplement 1A, Figure 2-figure supplement 1C). SMC2-mAID-GFP was expressed at a level similar to the endogenous SMC2 of wild type cells (Figure 1C) and localised correctly along the axis of sister chromatids in living cells (Figure 1D). Prometaphase chromosomes of SMC-AID-GFP cells appeared indistinguishable from those of wild type cells in the absence of auxin (Figure 1G, Figure 1-figure supplement 1B).

\section{Chromosomes condense into a single mass following rapid SMC2 depletion}

Auxin addition induced the rapid depletion of SMC2-mAID-GFP in asynchronous cell cultures. After a $3 \mathrm{~h}$ auxin treatment, SMC2-mAID-GFP fell to approximately $5 \%$ of the wild-type level, the number of GFP-positive cells declined, 
113 and the GFP signal was lost (Figure 1C, E, Figure 1-figure supplement 1C). Chromatin

114 of mitotic cells depleted of SMC2 clustered in a compact mass in which single

115 chromosomes could not be resolved (Figure 1E-G, Figure 1-figure supplement 1B).

116 The chromosome morphological defects seen in SMC2-AID-GFP cells treated with

117 auxin were much more severe than those in SMC2 ${ }^{\text {OFF }}$ cells treated with doxycycline

118 for 30-34 h. SMC2 ${ }^{\text {OFF }}$ (conventional conditional knockout) chromosomes were wider

119 and fuzzier than controls, but individual chromosomes were still visible (Figure 1G,

$1202 \mathrm{Ai}$, Figure 1-figure supplement $1 \mathrm{~B})$. Furthermore, SMC2 ${ }^{\text {OFF }}$ cells underwent aberrant

121 anaphase with chromosome bridges as previously reported (Figure 1Fiv). In previous

122 reports, chromosome mis-segregation at anaphase was the most prominent

123 phenotypic defect after condensin depletion or inactivation (Saka et al., 1994;

124 Steffensen et al., 2001; Hagstrom et al., 2002; Hudson et al., 2003). In contrast, we

125 were surprised to observe no anaphase or telophase cells following rapid SMC2

126 depletion (Figure 2C).

127 The strong chromosome phenotype observed after rapid condensin depletion

128 made it difficult to determine the stage of mitosis at which the cells arrested. We

129 therefore stained a sub-clone of SMC2-AID-GFP cells, expressing CENP-H-GFP from

130 the endogenous allele, with antibodies to histone H3S1Oph and INCENP following

131 treatment with vehicle (ethanol) or auxin for $3 \mathrm{~h}$. The signal intensity of H3S10ph is

132 normally high on prometaphase and metaphase chromosomes and progressively

133 decreases after anaphase onset. INCENP is a scaffolding and regulatory subunit of

134 the chromosome passenger complex that localises on chromosome arms and

135 centromeres in early mitosis, then transfers to the central spindle and cleavage

136 furrow during mitotic exit (Carmena et al., 2012).

137 The H3S10ph level on the SMC2-depleted chromosome mass resembled that

138 on control prometaphase or metaphase chromosomes (Figure 1Ei,iii, 2Aii,iii).

139 Furthermore, INCENP localised on the SMC2-depleted chromosome mass and not on

140 the spindle (Figure 2-figure supplement $1 \mathrm{~A}$ ). These observations suggest that after

141 auxin treatment, SMC2-depleted cells with chromosome clusters were in early

142 mitosis.

143 These results in SMC2-AID-GFP cells treated with auxin revealed that

144 condensin is required to shape and resolve individual mitotic chromosomes in 
145 cultured vertebrate cells. In addition, phenotypic differences between SMC2 ${ }^{\text {OFF }}$ cells

146 and SMC2-AID-GFP cells depleted of SMC2 supported our hypothesis that the milder

147 defects observed in previously reports were due to the gradual loss of condensin.

148

Use of chemical genetics to obtain mitotically synchronised cells lacking condensin

In order to obtain DT40 cultures progressing through mitosis in a

151 synchronous wave, we introduced a CDK1 ${ }^{\text {as }}$ CDNA into SMC2-AID-GFP cells and then

152 disrupted the endogenous CDK1 alleles using CRISPR/Cas9 technology. 1NMPP1, a

153 bulky ATP analogue, inhibits the mutant CDK1 ${ }^{\text {as }}$ but no other kinases in these cells.

154 As expected, 1NMPP1 did not inhibit cells from progressing through $\mathrm{G}_{1}, \mathrm{~S}$ and $\mathrm{G}_{2}$

155 phases, however, it prevented $\mathrm{G}_{2}$ cells from entering mitosis, and forced pre-existing

156 mitotic cells to exit mitosis prematurely (Hochegger et al., 2007). No mitotic cells

157 were detected 30 min after addition of 1NMPP1 (Figure 2-figure supplement 1B).

158 Subsequent washout of 1NMPP1 from the arrested $\mathrm{G}_{2}$ cells triggered a rapid and

159 near synchronous wave of mitotic entry and exit (Figure 2C, Figure 2-figure

160 supplement $1 \mathrm{C})$.

161 Introduction of the CDK1 ${ }^{\text {as }}$ circuit into SMC2-AID-GFP cells (termed SMC2-

162 AID-GFP/CDK1 ${ }^{\text {as }}$ cells) allowed us to investigate the effects of depleting condensin

163 either just prior to mitosis or during a mitotic arrest while avoiding confounding

164 effects from previous cell divisions.

165 A typical experimental time line using SMC2-AID-GFP/CDK1 ${ }^{\text {as }}$ cells is

166 illustrated in Fiure 2B (see Materials and Methods). All control cells (no auxin) were

167 in interphase at $\mathrm{T}=0 \mathrm{~min}$. The mitotic index of the control cells increased gradually

168 from $\mathrm{T}=15 \mathrm{~min}$, peaked at $\mathrm{T}=60 \mathrm{~min}$, then decreased thereafter (Figure $2 \mathrm{C}$ ).

169 Prophase cells were observed at $\mathrm{T}=15 \mathrm{~min}$ but were mostly gone by $30 \mathrm{~min}$. Most

170 mitotic cells at $\mathrm{T}=30-60$ min were in prometaphase or metaphase (Figure $2 \mathrm{C}$ ). Cells

171 in anaphase and telophase were seen at $\mathrm{T}=60-120 \mathrm{~min}$. Furthermore, $\mathrm{G} 1$ cells

172 were detected $1 \mathrm{~h}$ after release from a 1NMPP1 block by DNA content analysis

173 (Figure 2-figure supplement 1C). Thus, mitosis lasts about one hour in control cells

174 after release from a $4 \mathrm{~h}$ 1NMPP1 block.

175 
176 SMC2-depleted cells accumulate at abnormal prometaphase then exit mitosis

177 without segregating chromosomes

178 Control cells exhibited prophase chromosome condensation within 15 min of

179 the 1NMPP1 washout (Figure 2C). In contrast, SMC2-depleted cells did not show

180 chromatin condensation until after nuclear envelope breakdown, as previously

181 reported with RNAi or conditional knockouts of condensin (Hudson et al., 2003;

182 Hirota et al., 2004; Ono et al., 2004).

183 The lack of prophase condensation could explain the slightly lower mitotic

184 index of SMC2-depleted cells at T= $15 \mathrm{~min}$ (Figure $2 \mathrm{C}$ ). However, the mitotic index

185 caught up with that of control cells by $T=30-60$ min and continued to increase

186 throughout the time course. No recognizable anaphase or telophase was observed in

187 SMC2-depleted cells over the $2 \mathrm{~h}$ time course of this experiment confirming the

188 results in asynchronous culture (Figure 2C, D). Nevertheless huge polyploid

189 interphase and mitotic cells were seen following 2 days auxin treatment (Figure 2-

190 figure supplement $1 \mathrm{E}$ ), suggesting somehow these cells exited the previous mitosis.

191 Live cell imaging of SMC2-depleted cells showed that these cells exited

192 mitosis without properly segregating chromosomes (Figure 2E) although control cells

193 did (Figure 2-figure supplement 1D). This can explain why we did not observe

194 anaphase or telophase cells following SMC2-depletion.

195

196

SMC2-depleted cells have a malformed mitotic spindle with the chromosome mass

to one side

Control chromosomes congress to the middle of a bipolar spindle during late prometaphase and metaphase (Figure 1E, 2Di, 3A, 3B, Figure 3-figure supplement movie 1). In contrast, the single amorphous mass formed by SMC2-depleted chromosomes appeared to be largely excluded from the spindle. A few chromatin

202 fibers protruded from this mass and extended into the spindle with centromeres at

203 their tips (Figure 2Di, 3A, Figure 3-figure supplement movie 2). This suggests that

204 chromosome arms did not follow as their centromeres congressed to the spindle 205 midzone.

206 The mitotic spindle in SMC2-depleted cells was bipolar but often twisted or 207 asymmetric (Figure 1E, 2D, 3A, 3B, Figure 3-figure supplement movie 2). Mitotic 
208 spindles and chromosomes in SMC2-depleted cells did not position properly

209 between the two centrosomes, which were visualised using PACT-RFP

210 (pericentrin/AKAP450 centrosomal targeting - Figure 3A, B). In order to quantify the

211 relative positions of chromosomes relative to the mitotic spindles, angles between

212 chromosomes and centrosomes in late prometaphase or metaphase cells were

213 measured using SMC2-AID-GFP/CDK $1^{\text {as }}$ cells expressing PACT-RFP (see Materials and

214 Methods). The angle between the centre of mass of the chromosomes and the two

215 centrosomes was between 155-180 degrees in most control cells (18 out of 22)

216 (Figure 3B,C). In contrast, this angle was less than 90 degrees in nearly half of the

217 SMC2-depleted cells (13 out of 27).

218 Forces exerted by spindle attachments exacerbated the structural defects of

219 mitotic chromosomes and altered the relative position and shape of the

220 chromosomes and spindle. When microtubules were depolymerized by nocodazole

221 treatment in control cells, rod-shaped individual mitotic chromosomes could be

222 recognized (Figure 3D). In contrast, individual chromosomes could not be resolved in

223 SMC2-depleted cells treated with nocodazole although the chromosome mass

224 became more symmetrical and protruding chromatin fibers disappeared.

225 CENP-I is a kinetochore protein whose depletion by RNAi causes defects in

226 kinetochore assembly, visualized as decreased CENP-T levels at kinetochores (Figure

$2273 \mathrm{E}-\mathrm{G})$. Microtubules do not bind tightly to such compromised kinetochores

228 (Nishihashi et al., 2002; Hori et al., 2008a). In CENP-I-depleted cells, the

229 morphologies of SMC2-depleted chromosomes were still aberrant, however the

230 chromosome mass was symmetrical and overlapped the mitotic spindle, which also

231 adopted a symmetrical shape (Figure 3G).

232 These experiments confirm that condensin is required to shape mitotic

233 chromosomes and reveal that mitotic spindle forces further deform the amorphous

234 SMC2-depleted chromosomes, presumably because SMC2-depleted chromosomes

235 lack structural integrity in arms and in pericentromere regions as reported previously

236 (Oliveira et al., 2005; Gerlich et al., 2006; Bouck and Bloom, 2007; Ribeiro et al.,

237 2009). We also confirmed that lack of structural integrity in SMC2-depleted

238 chromosomes results in mispositioning of the chromosome mass and spindle

239 malformations (Wignall SM 2003, Ono T 2004). 
SMC2-depleted kinetochores fail to make stable attachments to microtubules

The CENP-H-GFP signals on SMC2-depleted kinetochores that stretched out

243 from the chromosome mass were round ( 21 out of 21 centromeres) and resembled

244 those in control metaphase cells, where correlative light and electron microscopy

245 (CLEM) revealed a typical trilaminar kinetochore structure with attached

246 microtubules (Figure 4A arrows, B). In contrast, the CENP-H-GFP signal on SMC2-

247 depleted kinetochores inside or at the edge of the chromosome mass was often

248 deformed (Figure 4C yellow arrowheads, see also Figure 2A and Figure 3-figure

249 supplement movie 2). Indeed the trilaminar structure was less evident following

250 SMC2 depletion (Figure 4A, B). The shape of kinetochores may be distorted due to

251 lack of structural integrity of the underlying chromatin as previously suggested

252 (Samoshkin 2009).

253 Strong MAD2 signals were seen in almost all SMC2-depleted late

254 prometaphase cells ( 24 out of 25 cells at $1 \mathrm{~h}$ after release from $4 \mathrm{~h} 1 \mathrm{NMPP} 1$ block)

255 (Figure 4C). In contrast, approximately half of control late prometaphase cells

256 showed strong MAD2 signals (13 out of 25 cells) and other cells showed only weak

257 MAD2 signal (11 out of 25 cells). This suggested that these MAD2 positive

258 centromeres lack stable bipolar attachments and that the prometaphase

259 accumulation of SMC2-depleted cells (Figure 2C) might be due to an unsatisfied

260 spindle assembly checkpoint (SAC).

261 Indeed, silencing of the SAC by Mad2 RNAi caused both SMC2-depleted and

262 control cells to enter anaphase with unaligned chromosomes (Figure 4-figure

263 supplement 1). Mitotic exit was confirmed by INCENP staining on the central spindle

264 and by reduced Cyclin B2 staining (Figure 4-figure supplement $1 \mathrm{~A}, \mathrm{Bi}$,iii,iv).

265 Approximately 80\% of SMC2-depleted cells treated with MAD2 siRNA showed highly

266 uneven chromosome segregation with chromosome bridges and centromeres

267 trapped in the intercellular bridge (Figure 4-figure supplement 1A, B, D). Live cell

268 imaging showed that all SMC2-depleted daughter cells treated with MAD2 siRNA

269 fused after an abortive cytokinesis (Figure 4-figure supplement 1E).

270 Thus, SMC2-depleted kinetochores can attach to microtubules but are

271 defective in forming stable amphitelic attachments. 
273 SMC2 is required for intrinsic chromosome organization but not for mitotic

\section{4 chromatin compaction}

275 To better understand the consequences of rapid SMC2 depletion on

276 chromosome morphology we performed an ultrastructural analysis using 3D-CLEM

277 with SBF-SEM (serial block face scanning electron microscopy) and digital

278 reconstructions (Booth et al., 2016).

279 To establish a default set of control parameters, the parental wild type DT40

280 cell line was first analysed. The entire chromosome complement of a metaphase

281 control cell, was reconstructed from 140 serial sections and modeled using Amira

282 (Figure 5A). The presence of two pairs of centrioles surrounded by PCM and flanking

283 the aligned chromosomes confirmed that the cell was indeed in metaphase. Using

284 separation algorithms we identified 74 discrete chromosome units (Figure 5Av-vi),

285 with a combined volume of $75 \mu^{3}$ and surface area of $424 \mu \mathrm{m}^{2}$ (Figure 5D). The

286 normal DT40 karyotype has 80 chromosomes, though scoring of the smallest

287 microchromosomes can be difficult (Sonoda et al., 1998; Molnar et al., 2014).

288 A similar analysis was next performed on a cell following rapid SMC2

289 depletion (Figure 5B). Remarkably, despite the clear disorganization of the chromatin

290 mass, it was possible to resolve 84 chromosome units (Figure 5C), with a combined

291 volume of $71 \mu \mathrm{m}^{3}$ and surface area of $931 \mu \mathrm{m}^{2}$ (Figure 5D). Interestingly, although

292 both the chromosome number and combined volumes were similar (74 vs 84 and 75

$293 \mu \mathrm{m}^{3}$ vs $71 \mu^{3}$ ), the surface area had more than doubled following SMC2 depletion

$294\left(424 \mu \mathrm{m}^{2}\right.$ vs $\left.931 \mu \mathrm{m}^{2}\right)$. This suggests that chromatin compaction measured as total

295 chromatin volume was near normal, but that the shape of the chromosomes as

296 revealed by the surface area was severely compromised. By ranking the

297 chromosomes according to their volumes, we could confirm that individual

298 chromosomes from the depleted cell had a similar volume, but a far greater surface

299 area than wild-type chromosomes (Figure 5D).

300 This analysis confirms that, as previously suggested (Vagnarelli et al., 2006),

301 condensin has a key role in establishing a normal mitotic chromosome architecture,

302 but is not essential for volume-wise reduction of chromatin (chromatin compaction)

303 from interphase to mitosis. 
305 Condensin is essential for normal intrinsic architecture of condensed mitotic 306 chromosomes

307 Use of the intrinsic mitotic chromosome structure (IMCS) assay previously

308 established that condensin is required to establish a normal mitotic chromosome 309 architecture (Hudson et al., 2003) (Figure 6A). In this assay, cells were treated with

310 nocodazole for $12 \mathrm{~h}$ before exposure to a hypotonic buffer (Figure 6-figure

311 supplement $1 \mathrm{~A})$. To test condensin's role in establishing or maintaining the structural

312 memory of mitotic chromosomes, respectively, cells were treated with auxin either

313 for the entire $12 \mathrm{~h}$ (SMC2 depleted before mitotic chromosome assembly) or for the

314 last $2 \mathrm{~h}$ (SMC2 depleted after mitotic chromosome assembly). Auxin was omitted

315 from controls so that SMC2-mAID-GFP was continuously present.

316 For the IMCS assay, chromosomes were subjected to two cycles of unfolding

317 and refolding, then their shape was scored in one of three categories: reformed,

318 partially reformed or disorganised (Figure 6A, B). Approximately $90 \%$ of control cells

319 (minus auxin) showed either reformed or partially reformed chromosomes (Figure

$3206 \mathrm{~B}, \mathrm{C}$ ). In contrast, cells depleted of SMC2 prior to chromosome assembly (auxin for

$32112 \mathrm{~h}$ ) exhibited only disorganized chromatin after this treatment. Remarkably, a

322 similar result was obtained for $>90 \%$ of cells depleted of SMC2 only after mitotic

323 chromosome formation (auxin for $2 \mathrm{~h}$ ). Depletion of SMC2-mAID-GFP after auxin

324 addition was confirmed by immunoblot analysis (Figure 6-figure supplement 1B, C).

325 Thus, the structural memory of mitotic chromosomes is not maintained in the

326 absence of condensin, even when SMC2 is degraded after mitotic chromosomes

327 have formed.

328 We next exploited the ability of SMC2-AID-GFP/CDK1 ${ }^{\text {as }}$ cells to enter mitosis

329 in a synchronous wave in order to follow the process of chromosome formation in

330 the presence or absence of condensin. We also characterized chromosomes

331 depleted of SMC2 during mitosis (Figure 6D, Figure 6-figure supplement 2A).

$332 \quad$ All cells in the culture were in interphase at the end of a $13 \mathrm{~h}$ 1NMPP1

333 treatment $(T \approx 0.5 \mathrm{~h}$ ) (Figure $6 \mathrm{E}$ ). Approximately $80 \%$ of those cells entered mitosis

334 within $1 \mathrm{~h}$ after $1 \mathrm{NMPP} 1$ washout $(\mathrm{T}=1.5 \mathrm{~h})$. The mitotic index then remained almost

335 unchanged until nocodazole washout ( $T=4.5 \mathrm{~h})$. 

treatment (Figure 6Ei, 6Gi: T=1.5 $-4.5 \mathrm{~h}$ ). Chromosomes began to align at a metaphase plate by 30 minutes after nocodazole washout at $\mathrm{T}=4.5 \mathrm{~h}$ (Figure 6Ei). Most of these cells exit mitosis at $T=\leq 6 \mathrm{~h}$, as revealed by a reduction in Cyclin B2 levels (Figure 6-figure supplement 2C, Ei, Fi). condensin is required to maintain chromosome morphology in mitosis. Rod-shaped chromosomes were clearly visible prior to auxin addition at $\mathrm{T}=1.5 \mathrm{~h}$, but became much less distinct at $\mathrm{T}=2.5 \mathrm{~h}$, within $1 \mathrm{~h}$ of auxin addition (Figure 6Eii). After hypotonic treatment, chromosomes appeared normal at $\mathrm{T}=1.5 \mathrm{~h}$, but were completely disorganised within $1 \mathrm{~h}$ of auxin addition at $\mathrm{T}=2.5 \mathrm{~h}$ (Figure 6Gii, 6Hii). chromosome structure was defective at all time points following release from the 1NMPP1 block ( $T=1.5-4.5 \mathrm{~h}$ - Figure 6Eiii). Without hypotonic treatment, these SMC2-depleted mitotic chromosomes appeared as a condensed ball. In cells treated with hypotonic buffer prior to fixation, SMC2-depleted mitotic chromosomes formed

352 a disorganized mass of dispersed chromatin at all time points (Figure 6Giii, 6Hiii).

353 The morphology of SMC2-depleted mitotic chromosomes and subsequent 354 chromosome mis-segregation defects were indistinguishable regardless of whether 355 SMC2 was depleted prior to mitotic entry or during mitosis after chromosomes had 356 formed. This confirms for somatic cells the early observation (Hirano and Mitchison, 357 1994) and recent studies with mouse oocytes and Drosophila embryo (Houlard 2015,

358 Piskadlo 2017), that condensin is necessary to maintain the structure of mitotic chromosomes throughout mitosis.

\section{Titration of condensin levels reveals differential requirements for SMC2 in mitotic}

\section{chromosome formation and chromosome segregation}

364 for sister chromatid segregation at anaphase. To further explore the functional link

365 between these two processes, we used auxin treatments to produce cultures with a

366 graded series of condensin concentrations. This was possible because the abundance 
367 of an AID tagged protein can be manipulated by varying the auxin concentration

368 (Nishimura et al., 2009).

Auxin concentrations yielding a range of partial depletions of SMC2-mAID-

370

371

372

373

374

375

376

377

378

379

380

381

382

383

384

385

386

387

388

389

390

391

392

393

394

395

396

397 GFP were selected based on data obtained from flow cytometry, microscopy, and immunoblotting analysis (Figure 7A, B). SMC2-AID-GFP/CDK1 ${ }^{\text {as }}$ cells were treated with 1NMPP1 for $4 \mathrm{~h}$ and with various concentrations of auxin for $3 \mathrm{~h}$. After release from 1NMPP1 to allow entry into mitosis, cells were fixed every $30 \mathrm{~min}$ and analysed as in Figure 2. Auxin was omitted from wash and release media apart from 125/250 $\mu \mathrm{M}$ auxin treated cells because we noticed that in mitotic cells even low auxin concentrations eventually resulted in depletion levels equivalent to those seen with $125 / 250 \mu \mathrm{M}$ auxin treatment. This is likely due to the reduction of protein synthesis during mitosis, such that even a low degradation activity could eventually deplete the AID-tagged protein.

Immunoblot analysis showed that cellular levels of SMC2 fell to 75\% or $\sim 45 \%$ of those in wild-type cells after addition of $1.95 \mu \mathrm{M}$ or $7.81 \mu \mathrm{M}$ of auxin, respectively (Figure 7A). (The absolute levels vary between experiments but the relative levels are preserved.) Reduction of SMC2 by $25 \%$ had little impact on either the morphology or segregation of mitotic chromosomes (Figure 7C). However, chromosome segregation defects were observed when SMC2 fell to $55 \%$ of wild type levels. In those cells, prometaphase chromosomes were slightly wider but individual rod-shaped chromosomes were still discernible. Despite this near normal morphology, some chromatin bridges were observed when the cells entered anaphase and telophase.

We next asked whether the observed defects in sister chromatid segregation were due to defects in the intrinsic structure of the chromosomes or to another function of condensin. We exposed SMC2-AID-GFP/CDK1 $1^{\text {as }}$ cells to 1 NMPP1 for $4 \mathrm{~h}$ plus various concentrations of auxin for $3 \mathrm{~h}$, then washed out the 1NMPP1 to allow cells to enter mitosis (Figure 8A). At 45 min after 1NMPP1 washout (when cells were in prometaphase), cells were subjected to the IMCS assay as in Figure 6A. Later, at 90 min after 1NMPP1 washout (when cells were in anaphase or telophase), cells were fixed and stained to examine sister chromatid segregation. For cells treated with 125 
$398 \mu \mathrm{M}$ auxin we did not observe typical anaphase or telophase cells, so we instead

399 scored chromatin bridges at cytokinesis.

$400 \quad$ This experiment revealed very clear differences in the threshold

401 requirements for condensin in chromosome architecture and sister chromatid

402 segregation. In the IMCS assay, mitotic chromosome structures reformed in $90 \%$ of

403 cells when SMC2 protein levels had fallen to $60 \%$ of wild type (15.63 $\mu \mathrm{M}$ auxin -

404 Figure 8B, D). In contrast, only $45 \%$ of cells successfully segregated chromosomes

405 without chromosome bridges under these conditions (Figure 8C, D).

406 Collectively, these results reveal that higher levels of condensin are required

407 for sister chromatid resolution and anaphase chromosome segregation than for

408 formation of a normal mitotic chromosome architecture, as assayed by the IMCS

409 assay.

\section{Partial depletion of condensin disrupts the chromosome axes}

412 Use of the auxin titration series revealed that even under conditions of

413 partial condensin depletion where the IMCS assay reports that chromosome

414 architecture is near normal, the organization of the chromosome axes is strongly

415 affected. When condensin levels have been depleted by as little as $50 \%$, the

416 distribution of condensin and topo Il $\alpha$ is disrupted and the proteins appear to diffuse

417 throughout the chromosomes. This cannot be observed in $125 \mu \mathrm{M}$ auxin, where

418 condensin is effectively gone and topoisomerase Il $\alpha$ is no longer visible on the

419 chromatid (Figure 9A). Diffuse chromosomal localisation of topoisomerase lla has

420 been reported previously following conventional condensin depletion (Hudson 2003,

421 Coelho 2003). The anaphase bridges observed under partial depletion of SMC2 could

422 be explained by incomplete decatination and/or possibly recatination of sister

423 chromatids due to disruption of chromosome axis and diffuse localisation of

424 topoisomerase Il $\alpha$ as recently reported (Piskadlo 2017). Furthermore, it could explain

425 why the chromosome segregation defects have been observed as the most

426 prominent and universal defect when condensin depletion occurred with slower

427 kinetics.

428 
429

430

431

432

433

434

435

436

437

438

440

\section{DISCUSSION}

Mitotic chromosome condensation is a complex process, involving removal of interphase chromatin structures (TADs and compartments), two-to-three-fold compaction of the chromatin, formation of rod-shaped structures and sister chromatid resolution (Vagnarelli, 2012; Naumova et al., 2013). The role(s) of condensin in these processes remain unclear. Here we show that condensin is not required for mitotic chromatin volume compaction and that different threshold levels of condensin are required for establishment of mitotic chromosome architecture and for chromatid segregation in anaphase.

There has been a long-standing controversy as to what are the primary mitotic defects after condensin depletion. Prior to the discovery of condensin, it was reported that SMC proteins were needed to both assemble and maintain mitotic chromosomes in Xenopus egg extracts (Hirano and Mitchison, 1994). A recent study of mouse meiotic cells and Drosophila embryos reached a similar conclusion (Houlard et al., 2015), but in between, many studies of somatic cells reported that rod-shaped chromosomes could form even when condensin had been significantly depleted (Bhat et al., 1996; Steffensen et al., 2001; Hagstrom et al., 2002; Somma et al., 2003; Hudson et al., 2003; Coelho et al., 2003; Vagnarelli et al., 2006; Maddox et al., 2006; Samoshkin et al., 2009). Interestingly, those studies agreed that although chromosomes could form in cells depleted of condensin subunits, anaphase sister chromatid separation was seriously impacted and prominent chromatin bridges were commonly seen.

The present study combined use of auxin inducible degron (AID) technology for rapid depletion of condensin with $\mathrm{CDK} 1^{\text {as }}$ alleles to obtain improved synchrony of mitotic entry. SMC2-AID-GFP/CDK1 ${ }^{\text {as }}$ cells showed more homogenous and more severe defects than those seen in SMC2 ${ }^{\text {OFF }}$ conditional knockout cells. Interestingly, residual levels of SMC2 were similar in both systems (5-7 \% of wild type cells). Importantly, the AID system appears to function particularly well in somatic mitotic cells, where protein translation is reduced. Thus, the residual levels of condensin in mitotic cells may be lower compared to those in G2 phase.

We hypothesize that previous discrepancies in chromosome morphology observed with differing experimental protocols did not simply reflect the extent of 
461 condensin depletion, as previously speculated, but instead the time taken to deplete

462 condensin, and the time allowed for cells to up-regulate compensatory pathways.

463 Use of SMC2-AID-GFP/CDK $1^{\text {as }}$ cells allowed us to obtain $>10^{8}$ cells with $\geq 80 \%$

464 purity in $\mathrm{G} 2$, prophase and prometaphase without microtubule inhibitors. We could

465 distinguish the roles of condensin at mitotic entry, during metaphase and during

466 mitotic exit because SMC2 could be depleted just prior to mitotic entry and also

467 within mitosis in a time restricted manner. We found that mitotic chromosomes

468 depleted of condensin after their assembly were morphologically and structurally

469 indistinguishable from chromosomes assembled in the absence of condensin. This is

470 consistent with observations in the Xenopus egg extract system (Hirano and

471 Mitchison, 1994). It is tempting to speculate that one essential role of condensin is

472 to lock-in the architecture of mitotic chromosomes and prevent other factors such as

473 topoisomerase Il $\alpha$ from promoting aggregation or random (de)catenation of the

474 chromatin.

475 Two recent studies showed that meiotic chromosomes in mouse oocytes or

476 mitotic chromosomes in early Drosophilia embyos rapidly depleted of condensin lost

477 structural integrity and were morphologically aberrant (Houlard et al., 2015)

478 (Piskadlo 2017). Importantly in both of these studies and in the present work, the

479 resulting chromatin did not look like interphase, but was highly compacted although

480 the usual rod-like morphology was lost. The three studies thus clearly show that

481 condensin is essential for shaping and maintaining individual mitotic chromosomes

482 in vivo in embryos and in somatic cells. These results are consistent with the original

483 studies with Xenopus egg extract systems and with studies in S. pombe (Saka et al.,

484 1994). Thus, this role of condensin is evolutionally conserved.

485 In cells rapidly depleted of SMC2, individual chromosomes could not be

486 distinguished by conventional fluorescence microscopy within the single mass of

487 mitotic chromatin. Indeed, the first impression from conventional electron

488 microscopy was of a shattered mass of condensed chromatin. However, ultra-

489 structural analysis using 3D-CLEM with SBF-SEM and digital reconstructions (Booth et

490 al., 2016) successfully resolved the chromosome mass into units that apparently

491 correspond to individual chromosomes. Moreover, the total volume of SMC2- 
492 depleted chromosomes was similar to that of wild type chromosomes at metaphase

493 even though the surface area was $>4$-fold greater.

494 This result confirmed our previous hypothesis that condensin is dispensable for 495 mitotic chromatin volume-wise compaction, but required for structural organization

496 of the condensed chromosomes (Vagnarelli et al., 2006; Samejima et al., 2012).

497 Surprisingly, mitotic chromosomes can still form after conventional depletion of all

498 the three major components of the mitotic chromosome scaffold (condensin, 499 topoisomerase Il $\alpha$ and KIF4A) (Samejima et al., 2012). Furthermore, extensive 500 proteomic studies failed to identify any other major structural components of 501 mitotic chromosomes with a likely role in promoting mitotic chromatin compaction 502 (Ohta et al., 2010; Samejima et al., 2015). These results and other studies suggest

503 that chromatin compaction in mitosis is mediated by factors intrinsic to the 504 chromatin, possibly including histone post-translational modifications (Markaki et al., 505 2009; Georgatos et al., 2009) (Zhiteneva et al, 2017).

506 In addition to the obvious effects on chromosome architecture, rapid depletion 507 of SMC2 led to spindle defects that included abnormal exclusion of chromosomes

508 from the mitotic spindle as well as the shape of the mitotic spindle itself. One

509 characteristic of condensin depletion is the presence of thin chromatin fibers as

510 centromeres stretch out from the bulk of the chromatin. Condensin is essential for

511 establishing the proper elasticity of centromeric heterochromatin, an important

512 determinant of kinetochore movements under tension (Ribeiro et al., 2009;

513 Samoshkin et al., 2009; Jaqaman et al., 2010). The stretched fibers correspond to

514 centromeric heterochromatin that becomes abnormally compliant after condensin

515 depletion. This heterochromatin is stretched by spindle forces acting on

516 kinetochores. Interfering with microtubule attachments at kinetochores restores, to

517 some extent, the chromosome morphology.

518 Regarding the shape of the spindle, it has long been known that tension 519 stabilises kinetochore-microtubule attachments (Nicklas and Koch, 1969; Akiyoshi et 520 al., 2010) (Miller MP et al, 2016). We postulate that either the lack of tension, 521 compromised kinetochore structure, or possibly mis-localised aurora B kinase 522 activity due to the loss of condensin affects kinetochore microtubule attachments.

523 Furthermore, lack of structural integrity in chromosomes appeared to cause their 
524 incorrect positioning relative to centrosomes and the mitotic spindle. It is tempting

525 to speculate that the centromeres are under force from both the mitotic spindle and

526 the bulky chromosome mass. This may in turn produce the abnormal mitotic spindle

527 morphology observed in SMC2-depleted cells and possibly promote incorrect

528 kinetochore-microtubule attachments (Saunders and Hoyt, 1992) (Wignall SM 2003,

529 Ono T 2004).

$530 \quad$ Availability of an AID degron allele allowed us to examine the effects of

531 creating a hypomorphic series in which levels of SMC2 were lowered to differing

532 extents. The residual amounts of AID tagged proteins are inversely correlated with

533 the auxin concentration (Nishimura et al., 2009). Use of a titration series allowed us

534 to demonstrate that higher levels of condensin are required for successful anaphase

535 sister chromatid separation than for establishing the metaphase chromosome

536 architecture as defined by our IMCS assay. In other experiments, cells rapidly

537 depleted of either condensin I or II complex alone still form morphologically distinct

538 individual mitotic chromosomes but exhibit chromosome segregation defects,

539 indicating the requirement for a higher amount of condensin at anaphase (data not

540 shown). This could explain why severe mitotic chromosome assembly defects were

541 not reported in the experimental systems with gradual depletion of condensin.

542 Failures in cytokinesis during previous cell divisions could have interfered with the

543 observation of morphological defects at the next mitosis.

544 Why is the threshold amount of SMC2 higher for segregating chromosomes?

545 Partially lowering condensin levels appears to disrupt the orderly distribution of

546 condensin and topo II $\alpha$ along the chromatid axes. This altered distribution of the two

547 proteins may either cause anaphase chromosomes to become mechanically more

548 fragile or interfere with the decatenation of sister chromatids. The existence of

549 different threshold levels of condensin is consistent with the idea that condensin's

550 roles in chromosome condensation and sister-chromatid separation are different

551 (Sullivan et al., 2004; D'Amours et al., 2004).

552 In summary, we show here that the architecture of mitotic chromosomes in

553 somatic cells is not static but must be maintained by the action of condensin

554 throughout mitosis. In the absence of condensin, chromatin appears to be fully

555 compacted in volume but lacks a normal architecture. Rigidity of mitotic 
556 chromosomes is essential for proper kinetochore and microtubule attachments and

557 also for correct shaping and positioning of the mitotic spindle. Wild-type levels of

558 condensin are particularly critical to ensure the completion of sister chromatid

559 separation in anaphase even though the bulk of sister chromatid resolution normally

560 occurs during prophase (Nagasaka et al., 2016).

561 


\section{MATERIALS AND METHODS}

563 Cell culture, transfection, siRNA

564 Chicken DT40 (B lymphoma) cells were cultured in RPMI1640 medium

565 supplemented with $10 \%$ fetal bovine serum and $1 \%$ chicken serum at $39^{\circ} \mathrm{C}$ in $5 \% \mathrm{CO}_{2}$

566 in air.

567 Stable transfection of DT40 cells was performed as described previously

568 (Samejima et al., 2008). We utilized Neon setting 5 (ThermoFisher Scientific) to

569 transiently transfect plasmid DNA (4-10 $\mu \mathrm{g})$ or siRNA oligonucleotides ( $2 \mu \mathrm{l}$ of 100

$570 \mu \mathrm{M}$ ) into 2-4 million cells suspended in $100 \mu$ l buffer $\mathrm{R}$ from the Neon kit. MAD2

571 siRNA duplex oligonucleotides were synthesized: (5'-

572 GAAAGCCAUUCAGGAUGAAAUUCGA $\left.-3^{\prime}\right)$ and (5'-

573 UCGAAUUUCAUCCUGAAUGGCUUUC -3') (ThermoFisher Scientific). Control

574 oligonucleotide (AllStars Negative Control siRNA) was purchased from QIAGEN.

575

576 Cell lines

SMC2 conditional DT40 knockout (SMC2 ${ }^{\text {ON/OFF }) ~ c e l l s: ~ S M C 2 ~}{ }^{\text {ON/OFF }}$ cells were

578 previously established (Hudson et al., 2003). In brief, the endogenous SMC2 gene

579 locus (exon 1-6 including the start codon) was disrupted by Histidinol resistance

580 cassette. These cells depend on the expression of a GgSMC2 CDNA driven by a

581 tetracycline suppressible promoter $\left(\mathrm{SMC2}^{\mathrm{ON}}\right)$. Addition of doxycycline stops the

582 expression of SMC2 protein. SMC2 protein was depleted in the cells treated with

583 doxycycline for $>30 \mathrm{~h}\left(\mathrm{SMC}^{\mathrm{OFF}}\right)$.

584 SMC2-AID-GFP DT40 cells: SMC2-AID-GFP cells were based on the SMC2 ${ }^{\text {OFF }}$ cells

585 which were constantly cultured in the presence of doxycycline $(0.5 \mu \mathrm{g} / \mathrm{ml})$ to so that

586 non-tagged GgSMC2 protein was not present in the cells. GgSMC2 cDNA fused with

587 minimal AID (mAID) tag (AtIAA17 ${ }^{65-132}$ ) (Natsume et al., 2016) and GFP tag (SMC2-

588 mAID-GFP) at the C-terminus was driven by a $3.8 \mathrm{~kb}$ fragment of the GgSMC2

589 promoter (Hudson et al., 2003) . SMC2-mAID-GFP was present as the sole source of

590 SMC2 protein in SMC2-AID-GFP cells (Figure 1A). A CMV promoter drove the

591 expression of plant specific F-box protein OsTIR1 linked to a MmDHFR cDNA by a T2A

592 peptide (synthesized at Thermofisher Scientific and cloned into pCDNA3). $10 \mu \mathrm{M}$

593 Methotrexate (MTX) was used to select cells expressing MmDHFR as well as OsTIR1 
594 at a high level. Plasmids coding SMC2-mAID-GFP and OsTIR1 were randomly

595 integrated into the genome.

$596 \quad C D K 1^{\text {as }}$ DT40 cells: CMV promoter drove the expression of the $\mathrm{XICdk} 1^{\text {as }}$ CDNA

597 (Hochegger et al., 2007) linked to a puromycin resistance gene by a T2A peptide. The

$598 \mathrm{GgCdk1}$ gene was inactivated by transient transfection of plasmids encoding hCas9

599 cDNA (addgene \#41815) and a GgCdk1 guideRNA (based on addgene \#41824). The

600 target sequence of the guide RNA was AAAATACGTCTAGAAAGTG.

601 Where desired, a CENP-H-GFP targeting vector (gift of Yasunari Takami,

602 University of Miyazaki) was used to tag the endogenous CENP-H locus in DT40 cell

603 lines. PACT-RFP construct is a gift of Viji Draviam (University of Cambridge).

604

605

\section{Antibodies and drug treatments}

606 Antibodies used for immunoblotting and indirect immunofluorescence analysis 607 were: rabbit anti-SMC2 (1:500) (Saitoh et al., 1994), guinea pig anti-Ggtopo $2 \alpha$

608 (1:1000) (Earnshaw et al., 1985), rabbit anti-GgCENP-T (1:1,000) (Hori et al., 2008b), 609 and rabbit anti-MAD2 (1: 100 gift of T. Fukagawa), rabbit-anti GgcyclinB2 (gift of Eric 610 Nigg), mouse anti- $\alpha$-tubulin B512 or DM1A (1:1,000-4,000; Sigma-Aldrich), mouse 611 anti-Cdk1 monoclonal A17 (1: 200-500: Abcam), and rabbit anti-histone H3 phospho 612 Ser 10 (D2C8) (1:1600, Cell Signaling).

613 Nocodazole dissolved in DMSO was added to a final concentration of $0.5 \mu \mathrm{g} / \mathrm{ml}$ 614 (Sigma-Aldrich). Doxycycline dissolved in water was added to a final concentration of $6150.5 \mu \mathrm{g} / \mathrm{ml}$ (BD) unless stated otherwise. 1NMPP1 dissolved in DMSO was added to a 616 final concentration of $2 \mu \mathrm{M}$. Indole-3-acetic acid (auxin) dissolved in ethanol was added to a final concentration of $125 \mu \mathrm{M}$ unless stated otherwise (Fluka).

\section{Use of CDK1as system}

620 Typically CDK1 ${ }^{\text {as }}$ cells were treated with $2 \mu \mathrm{M} 1 \mathrm{NMPP} 1$ for $4 \mathrm{~h}$ to accumulate 621 cells in $G_{2}$ phase. 1NMPP1 is a bulky ATP analogue that reversibly and specifically 622 inhibits analogue sensitive kinase mutants. 1NMPP1 treatment longer than $4 \mathrm{~h}$ 623 results in an increase of multipolar spindles due to centrosome amplification during

624 the $\mathrm{G}_{2}$ block. However, $\mathrm{CDK} 1^{\text {as }}$ cells blocked at $\mathrm{G}_{2}$ phase for more than $10 \mathrm{~h}$ could still 625 enter mitosis synchronously after 1NMPP1 washout without any apparent problems 
626 apart from the multipolar spindle. In order to release from the 1NMPP1 block, cells

627 were washed two to three times with fresh media.

628

\section{Indirect immunofluorescence of fixed cells}

Cells were fixed in pre-warmed $4 \%$ Formaldehyde/PBS for $10 \mathrm{~min}$ and permeabilized with $0.15 \%$ triton for 5-10 min. Cells were blocked with $5 \%$ BSA/PBS for $30 \mathrm{~min} .1^{\text {st }}$ antibody diluted in the blocking buffer was applied to the cells for $1 \mathrm{~h}$.

633 Cells were washed with PBS $3 x$ for 5 min. $2^{\text {nd }}$ antibody diluted in the blocking buffer

634 (1:500-1:1000, Alexa Fluor 488, 555 or 594, 647 (Molecular Probes, ThermoFisher

635 Scientific) was applied to the cells for $30 \mathrm{~min}$. Cells were washed with PBS for $5 \mathrm{~min}$

636 three times. DNA was stained with Hochest33452 and mounted with Prolong

637 diamond (Molecular Probes, ThermoFisher Scientific).

638 DeltaVision microscopy: 3D datasets were acquired using a cooled CCD camera (CoolSNAP HQ; Photometrics) on a wide-field microscope (DeltaVision Spectris; Applied Precision) with a 100× NA 1.4 Plan Apochromat lens. The datasets were deconvolved with softWoRx (Applied Precision), converted to Quick Projections in softWoRx, exported as TIFF files, and imported into Adobe Photoshop for final presentation.

3D structured illumination microscopy (3D-SIM): Super-resolution images were acquired using structured illumination microscopy. Samples were prepared on high precision cover-glasses (Zeiss, Germany). 3D SIM images were acquired on a NSIM (Nikon Instruments, UK) using a 100x 1.49NA lens and refractive index matched immersion oil (Nikon Instruments). Samples were imaged using a Nikon Plan Apo TIRF objective (NA 1.49, oil immersion) and an Andor DU-897X-5254 camera using 405, 488 and $561 \mathrm{~nm}$ laser lines. Step size for $Z$ stacks was set to 0.120 um as

651 required by manufacturers software. For each focal plane, 15 images (5 phases, 3

652 angles) were captured with the NIS-Elements software. SIM image processing,

653 reconstruction and analysis were carried out using the N-SIM module of the NIS-

654 Element Advanced Research software. Images were checked for artefacts using the

655 SIMcheck software (http://www.micron.ox.ac.uk/software/SIMCheck.php). Images

656 were reconstructed using NiS Elements software (Nikon Instruments) from a z stack

657 comprising at least $1 \mu \mathrm{m}$ of optical sections. In all SIM image reconstructions the 
658 Wiener and Apodization filter parameters were kept constant. 3D datasets were

659 visualized and analysed using Imaris V8.4 (Bitplane, Oxford Instruments, Oxfordshire, 660 UK).

661 Zeiss Airyscan microscopy: Images of chromosome scaffold proteins on 662 chromosome spreads were obtained using the Airyscan module on a Zeiss LSM 880

663 confocal, using a x100 alpha Plan-Apochromat objective. Hoechst 33342 was 664 detected using a $405 \mathrm{~nm}$ Diode laser and a 420-480 $\mathrm{nm}$ bandpass emission filter, 665 Alexa488 was detected using the $488 \mathrm{~nm}$ line of an Argon laser and a 495-550 nm 666 bandpass emission filter, the Alexa555 was detected using a HeNe561 laser and a $667570 \mathrm{~nm}$ long pass emission filter. Alexa647 was detected using a 633 laser and a 605 $668 \mathrm{~nm}$ long pass emission filter. Step size for $Z$ stacks was set to $0.145 \mu \mathrm{m}$. 3D datasets 669 were visualized and analysed using Imaris V8.4 (Bitplane, Oxford Instruments, 670 Oxfordshire, UK).

671

672 Live cell imaging with Zeiss Airy microscopy

673 Cells were centrifuged and suspended into Leibovitz's L-15 Medium (Thermo 674 Fisher Scientific) supplemented with 10\% FBS and 1\% Chicken serum. DNA was 675 visualised by $1 \mu \mathrm{M}$ SiR DNA (Spirochromome). GFP was detected using the $488 \mathrm{~nm}$ 676 line of an Argon laser and a 495-550 $\mathrm{nm}$ bandpass emission filter, RFP was detected 677 using a HeNe561 laser and a $570 \mathrm{~nm}$ long pass emission filter. SiR DNA was detected 678 using a 633 laser and a $605 \mathrm{~nm}$ long pass emission filter. Step size for $Z$ stacks was set 679 to $0.4 \mu \mathrm{m}$. Images were taken every $5 \mathrm{~min}$. 3D datasets were visualized and analysed 680 using Imaris V8.4 (Bitplane, Oxford Instruments, Oxfordshire, UK).

681

\section{Intrinsic mitotic chromosome structure (IMCS) assay}

Cells were enriched in mitosis either by $12 \mathrm{~h}$ nocodazole treatment $(0.5 \mu \mathrm{g} / \mathrm{ml})$

684 (Figure 6A-C) or by 1NMPP1 treatment (Figure 8A, B, D). Auxin was added at the

685 indicated time points (Figure $8 \mathrm{~A}$ ). The cells were plated on polylysine-coated slides

686 for $30 \mathrm{~min}$ before the following treatments. The slides were rinsed with PBS, 687 immersed in TEEN buffer for $5 \mathrm{~min}(1 \mathrm{mM}$ triethanolamine/ $\mathrm{HCl}, \mathrm{pH} 8.5,0.2 \mathrm{mM}$ $688 \mathrm{NaEDTA}$, and $25 \mathrm{mM} \mathrm{NaCl}$ ), before immersion into RSB buffer for $15 \mathrm{~min}$ (10 mM 689 Tris/ $\mathrm{HCl}, \mathrm{pH} 7.4,10 \mathrm{mM} \mathrm{NaCl}$, and $5 \mathrm{mM} \mathrm{MgCl}_{2}$ ). Following two cycles of TEEN 
690 buffer/RBS buffer treatments, the cells were fixed with 4\% Formaldehyde in RBS

691 buffer and mounted with Vectashield (containing DAPI). The final chromosome

692 morphologies were classified as reformed (green), partially reformed (yellow) or

693 disorganised (magenta).

694

\section{Quantitative immunoblotting}

696 Membranes were incubated with the relevant primary antibodies recognizing

$697 \alpha$-tubulin (as a loading control), SMC2, and MAD2, then subsequently with IRDye-

698 labeled secondary antibodies (LI-COR Biosciences). Fluorescence intensities were

699 determined using a CCD scanner (Odyssey; LI-COR Biosciences) according to the

700 manufacturer's instructions.

701

702

\section{Flow cytometry analysis}

703

GFP-positive and -negative living cells were analyzed using a FACSCalibur flow

704 cytometer following the manufacturer's instructions.

705 DNA contents: Cells were fixed with ice-cold $70 \%$ ethanol for overnight. These

706 cells were rinsed with PBS then resuspended with PBS containing $100 \mu \mathrm{g} / \mathrm{ml}$ RNAse A

707 and $5 \mu \mathrm{g} / \mathrm{ml}$ Propidium iodide and were analyzed using a FACSCalibur flow

708 cytometer following the manufacturer's instructions.

Measurement of the angle of chromosome mass and centrosomes

SMC2-AID-GFP/CDK1 ${ }^{\text {as }}$ cells expressing PACT-RFP was treated with 1NMPP1 for

$7124 \mathrm{~h}$ with/without a $3 \mathrm{~h}$ auxin treatment. $60 \mathrm{~min}$ after 1NMPP1 washout, cells were

713 fixed in pre-warmed $4 \%$ Formaldehyde/PBS for $10 \mathrm{~min}$. DNA was stained with

714 Hochest33452 and mounted with Prolong diamond (Molecular Probes, ThermoFisher

715 Scientific). Pictures of late prometaphase and metaphase cells were taken using Zeiss

716 Airy microscopy as described above. $(X, Y, Z)$ coordinates of the centre of

717 homogenous mass of PACT-RFP signals $\left(X_{1}, Y_{1}, Z_{1}\right)$ and $\left(X_{3}, Y_{3}, Z_{3}\right)$, and chromosomes

$718 \quad\left(X_{2}, Y_{2}, Z_{2}\right)$ were obtained using Imaris V8.4 (Bitplane, Oxford Instruments,

719 Oxfordshire, UK). The angle of chromosome mass and centrosomes was calculated

720 based on the coordinates. 
722

723

724

725

726

727

728

729

730

731

732

733

734

735

736

737

738

739

740

741

742

743

744

745

746

747

748

749

750

751

752

753

\section{SBF-SEM}

\section{Preparation of cells}

Cells were seeded onto gridded dishes (MatTek) and fixed with $3 \%$ glutaraldehyde and $1 \%$ paraformaldehyde in $0.1 \mathrm{M}$ sodium cacodylate buffer for $1 \mathrm{~h}$ at RT. Cells were then washed with PBS $3 \times 5$ min and samples prepared for SBF SEM (West et al., 2010). Extra contrasting steps were introduced, compared to those used for standard TEM to reduce charging and improve the signal to noise ratio. In detail, following fixation, the cells were post fixed and stained with reduced osmium, ( $2 \%$ osmium tetroxide in $\mathrm{dH}_{2} \mathrm{O}+1.5 \%$ Potassium Ferrocyanide in $0.1 \mathrm{M}$ sodium cacodylate buffer) for $1 \mathrm{~h}$ at RT. This was followed by $0.1 \%$ tannic acid in $\mathrm{ddd}_{2} \mathrm{O}$ for 20 min at RT. A second osmication step ( $2 \%$ in $\mathrm{ddH}_{2} \mathrm{O}$ for 40 min at RT), preceded an overnight incubation in aqueous $1 \%$ uranyl acetate at $4^{\circ} \mathrm{C}$. The next day cells were stained with Walton's lead aspartate $(0.02 \mathrm{M}$ in lead nitrate $+0.03 \mathrm{M}$ in aspartic acid in $\mathrm{dd}_{2} \mathrm{O}$, adjusted to $\mathrm{pH} 5.5$ ) for $30 \mathrm{~min}$ at $60^{\circ} \mathrm{C}$. To prevent precipitation artefacts the cells were washed for a minimum of $5 \times 3 \mathrm{~min}$ with $\mathrm{dd}_{2} \mathrm{O}$ between each of the staining steps described. Next, samples were dehydrated in a graded ethanol series of $30 \%, 50 \%, 70 \%, 90 \%$ in $\mathrm{ddH}_{2} \mathrm{O}$ for 5 min each, followed by $2 \times 5 \mathrm{~min} 100 \%$ ethanol. Samples were then infiltrated with TAAB Hard Premix resin at ratios of $1: 1$, 2:1 and 3:1 with resin: $100 \%$ ethanol, 30 min per incubation. Finally, samples were incubated in $100 \%$ resin for $2 \times 30$ min, before embedding the whole dish in $2 \mathrm{~mm}$ of $100 \%$ fresh resin. Samples were cured for $48 \mathrm{~h}$ at $60^{\circ} \mathrm{C}$.

\section{Preparation of blocks for 3View SBF-SEM}

Resin is separated from the gridded dish by trimming away the excess plastic and carefully sliding a razor between the dish and the resin (Booth et al., 2013). Excess resin is removed using a junior hacksaw and scalpel before the block is mounted onto a cryo-pin, cell side up using superglue. Targeted trimming is performed using an ultra-microtome and etched coordinates (Booth et al., 2013).

\section{SBF-SEM Imaging and Acquisition}

Samples were painted with Electrodag silver paint (avoiding the block face) and then coated with 10nm AuPd using a Q150T sputter coater (Quorum Technologies). The sample was inserted into the Gatan 3View sample holder and adjusted so the block face would be central in the microtome and parallel with the knife-edge. 
754 Consecutive sectioning and imagining was performed. To obtain a typical resolution

755 of $12 \mathrm{~nm}$ in $\mathrm{x}$ and $\mathrm{y}$, a frame width of $1024 \times 1024$ was used. Section thickness was 60

756 nm over $200-400$ sections.

$7573 D$ reconstruction, modeling and segmentation.

758 3View EM stacks were annotated using Amira (FEI). Chromosomes present in

759 every orthoslice were annotated using masking and thresholding alone (fully

760 automated) or in combination with magic wand and blow tools (semi automated).

761 The modelled complement of chromosomes was segmented into discernable

762 isolated objects using interactive thresholding and separate objects modules. Objects

763 were separated using 3D interpretation and a neighbourhood criteria of 26

764 connected elements, by at least one corner, edge or face. The marker contrast range

765 (H-extrema) was set between 5 and 7 , depending on the sample. Label analysis

766 modules were used to measure the geometry of all isolated structures. Surface

767 renders were generated using unconstrained smoothing at levels 5-7.

768

769

1NMPP1 Analogue Synthesis:

770

771

Synthesis of the ATP analog 4-amino-1-tert-butyl-3-(1'-

772 as described by (Hanefeld et al., 1996) with the following modifications. The starting

773 material was 1-naphthaleneacetic acid and the first two steps were carried out

774 under $\mathrm{N}_{2}$. In Step 2, triethylamine was used in place of $\mathrm{NaH}$ and the product 1-

775 naphthaleneacetyl malonylnitrile was recrystallized from acetonitrile and

776 chloroform. The enol ether product of Step 3 was purified on a silica gel column

777 (elution with 3:1 hexane:ethyl acetate) and stored under $\mathrm{N}_{2}$. The final product, a

778 white solid that gave a single spot on TLC, was purified similarly and further

779 recrystallized from ethyl acetate. Its identity was confirmed by ${ }^{1} \mathrm{H} N \mathrm{NMR}, \mathrm{HH}$ COSY

780 and high resolution mass spectrometry. ${ }^{1} \mathrm{H} \mathrm{NMR}\left(270 \mathrm{MHz}, \mathrm{CDCl}_{3}\right)$ gave peaks at 1.84

781 (singlet, 9H), 4.75 (singlet, 2H), 4.85 (broad singlet, 2H), 7.18 (doublet, 1H), 7.38

782 (triplet, $1 \mathrm{H}$ ), 7.54 (multiplet, 2H), 7.79-7.92 (multiplet, $2 \mathrm{H}$ ), 8.22 (doublet, $1 \mathrm{H}$ ) and

7838.24 (singlet, $1 \mathrm{H}$ ), in close agreement with published data (Bishop et al., 1999). Mass

784 spectrometry gave a mass of 331.181712 for the molecular ion (calculated mass for 
bioRxiv preprint doi: https://doi.org/10.1101/173633; this version posted August 9, 2017. The copyright holder for this preprint (which was not certified by peer review) is the author/funder. All rights reserved. No reuse allowed without permission.

$\left.785 \mathrm{C}_{20} \mathrm{H}_{21} \mathrm{~N}_{5}, 331.179696\right)$. The solid compound was stored at $4^{\circ} \mathrm{C}$. Aliquots were

786 dissolved in DMSO at $25 \mathrm{mM}$ and stored at ? $20^{\circ} \mathrm{C}$.

787 
788

789

790

791

792

793

794

795

796

797

798

799

800

801

802

803

804

805

806

807

808

\section{ACKNOWLEDGMENTS}

This work was funded by The Wellcome, of which W.C.E. is a Principal Research Fellow (Grant 107022). The Wellcome Centre for Cell Biology is supported by core funding from the Wellcome [203149]. M.T.K. was supported by JSPS KAKENHI Grants (No. 16K15095) and the JST PRESTO program. Sorting of GFP negative cells was performed in the Flow Cytometry facility, Institute of Immunology \& Infection Research, Edinburgh with assistance of Dr. Martin Waterfall. We thank Itaru Samejima for comments on the manuscript.

\section{Author contributions}

KS, experimental design, data acquisition, analysis and interpretation, wrote the manuscript; DB electron microscopy, manuscript revision; HO, MP and CW helped to make cell lines. JP and LX synthesized 1NMPP1; MK contribution of new experimental tools and manuscript revision; WCE, experimental design, data analysis and interpretation, co-wrote the manuscript.

\section{Conflicts of interest}

05 The authors declare that they have no competing interests. 
809

810

811

812

813

814

815

816

817

818

819

820

821

822

823

824

825

826

827

828

829

830

831

832

833

834

835

836

837

838

839

840

841

842

843

844

845

846

847

848

849

850

851

852

853

854

855

\section{REFERENCES}

Akiyoshi, B., K.K. Sarangapani, A.F. Powers, C.R. Nelson, S.L. Reichow, H. ArellanoSantoyo, T. Gonen, J.A. Ranish, C.L. Asbury, and S. Biggins. 2010. Tension directly stabilizes reconstituted kinetochore-microtubule attachments. Nature. 468:576-579.

Bhat, M.A., A.V. Philp, D.M. Glover, and H.J. Bellen. 1996. Chromatid segregation at anaphase requires the barren product, a novel chromosome-associated protein that interacts with Topoisomerase II. Cell. 87:1103-14.

Bishop, A., O. Buzko, S. Heyeck-Dumas, I. Jung, B. Kraybill, Y. Liu, K. Shah, S. Ulrich, L. Witucki, F. Yang, C. Zhang, and K.M. Shokat. 2000. Unnatural ligands for engineered proteins: new tools for chemical genetics. Annu Rev Biophys Biomol Struct. 29:577-606.

Bishop, A.C., C.Y. Kung, K. Shah, L. Witucki, K.M. Shokat, and Y. Liu. 1999. Generation of monospecific nanomolar tyrosine kinase inhibitors via a chemical genetic approach. Journal of the American Chemical Society. 121:627-631.

Booth, D.G., A.J. Beckett, O. Molina, I. Samejima, H. Masumoto, N. Kouprina, V. Larionov, I.A. Prior, and W.C. Earnshaw. 2016. 3D-CLEM Reveals that a Major Portion of Mitotic Chromosomes Is Not Chromatin. Mol Cell. 64:790-802.

Booth, D.G., L.P. Cheeseman, I.A. Prior, and S.J. Royle. 2013. Studying Kinetochore-Fiber Ultrastructure Using Correlative Light-Electron Microscopy. Microtubules, in Vitro, 2nd Edition. 115:327-342.

Bouck, D.C., and K. Bloom. 2007. Pericentric chromatin is an elastic component of the mitotic spindle. Curr. Biol. 17:741-748.

Carmena, M., M. Wheelock, H. Funabiki, and W.C. Earnshaw. 2012. The chromosomal passenger complex (CPC): from easy rider to the godfather of mitosis. Nat. Rev. Mol. Cell Biol. 13:789-803.

Coelho, P.A., J. Queiroz-Machado, and C.E. Sunkel. 2003. Condensin-dependent localisation of topoisomerase II to an axial chromosomal structure is required for sister chromatid resolution during mitosis. J. Cell Sci. 116:4763-4776.

D'Amours, D., F. Stegmeier, and A. Amon. 2004. Cdc14 and condensin control the dissolution of cohesin-independent chromosome linkages at repeated DNA. Cell. 117:455-469.

Earnshaw, W.C., B. Halligan, C.A. Cooke, M.M.S. Heck, and L.F. Liu. 1985. Topoisomerase II is a structural component of mitotic chromosome scaffolds. J. Cell Biol. 100:1706-1715.

Gasser, S.M., and U.K. Laemmli. 1986. Cohabitation of scaffold binding regions with upstream/enhancer elements of three developmentally regulated genes of D. melanogaster. Cell. 46:521-530.

Gasser, S.M., T. Laroche, J. Falquet, E. Boy de la Tour, and U.K. Laemmli. 1986. Metaphase chromosome structure. Involvement of topoisomerase II. J. Mol. Biol. 188:613-629.

Gassmann, R., P. Vagnarelli, D. Hudson, and W.C. Earnshaw. 2004. Mitotic chromosome formation and the condensin paradox. Exp Cell Res. 296:3542. 
856

857

858

859

860

861

862

863

864

865

866

867

868

869

870

871

872

873

874

875

876

877

878

879

880

881

882

883

884

885

886

887

888

889

890

891

892

893

894

895

896

897

898

899

900

901

902

903
Georgatos, S.D., Y. Markaki, A. Christogianni, and A.S. Politou. 2009. Chromatin remodeling during mitosis: a structure-based code? Front Biosci (Landmark Ed). 14:2017-27.

Gerlich, D., T. Hirota, B. Koch, J.M. Peters, and J. Ellenberg. 2006. Condensin I stabilizes chromosomes mechanically through a dynamic interaction in live cells. Curr. Biol. 16:333-344.

Hagstrom, K.A., V.F. Holmes, N.R. Cozzarelli, and B.J. Meyer. 2002. C. elegans condensin promotes mitotic chromosome architecture, centromere organization, and sister chromatid segregation during mitosis and meiosis. Genes Dev. 16:729-742.

Hanefeld, U., C.W. Rees, A.J.P. White, and D.J. Williams. 1996. One-pot synthesis of tetrasubstituted pyrazoles - proof of regiochemistry. J Chem Soc Perkin Trans 1. 1996:1545-1552.

Hirano, T. 2016. Condensin-Based Chromosome Organization from Bacteria to Vertebrates. Cell. 164:847-57.

Hirano, T., R. Kobayashi, and M. Hirano. 1997. Condensins, chromosome condensation protein complexes containing XCAP-C, XCAP-E and a Xenopus homolog of the Drosophila Barren protein. Cell. 89:511-521.

Hirano, T., and T.J. Mitchison. 1994. A heterodimeric coiled-coil protein required for mitotic chromosome condensation in vitro. Cell. 79:449-458.

Hirota, T., D. Gerlich, B. Koch, J. Ellenberg, and J.M. Peters. 2004. Distinct functions of condensin I and II in mitotic chromosome assembly. J. Cell Sci. 117:6435-45.

Hochegger, H., D. Dejsuphong, E. Sonoda, A. Saberi, E. Rajendra, J. Kirk, T. Hunt, and S. Takeda. 2007. An essential role for Cdk1 in S phase control is revealed via chemical genetics in vertebrate cells. J. Cell Biol. 178:257-268.

Hori, T., M. Amano, A. Suzuki, C.B. Backer, J.P. Welburn, Y. Dong, B.F. McEwen, W.H. Shang, E. Suzuki, K. Okawa, I.M. Cheeseman, and T. Fukagawa. 2008a. CCAN makes multiple contacts with centromeric DNA to provide distinct pathways to the outer kinetochore. Cell. 135:1039-1052.

Hori, T., M. Okada, K. Maenaka, and T. Fukagawa. 2008b. CENP-O class proteins form a stable complex and are required for proper kinetochore function. Mol. Biol. Cell. 19:843-854.

Houlard, M., J. Godwin, J. Metson, J. Lee, T. Hirano, and K. Nasmyth. 2015. Condensin confers the longitudinal rigidity of chromosomes. Nature Cell Biology. 17:771-+.

Hudson, D.F., P. Vagnarelli, R. Gassmann, and W.C. Earnshaw. 2003. Condensin is required for nonhistone protein assembly and structural integrity of vertebrate mitotic chromosomes. Dev Cell. 5:323-336.

Jaqaman, K., E.M. King, A.C. Amaro, J.R. Winter, J.F. Dorn, H.L. Elliott, N. McHedlishvili, S.E. McClelland, I.M. Porter, M. Posch, A. Toso, G. Danuser, A.D. McAinsh, P. Meraldi, and J.R. Swedlow. 2010. Kinetochore alignment within the metaphase plate is regulated by centromere stiffness and microtubule depolymerases. J. Cell Biol. 188:665-679.

Kanemaki, M.T. 2013. Frontiers of protein expression control with conditional degrons. Pflugers Arch. 465:419-25.

Lewis, C.D., and U.K. Laemmli. 1982. Higher order metaphase chromosome structure: evidence for metalloprotein interactions. Cell. 29:171-181. 
904

905

906

907

908

909

910

911

912

913

914

915

916

917

918

919

920

921

922

923

924

925

926

927

928

929

930

931

932

933

934

935

936

937

938

939

940

941

942

943

944

945

946

947

948

949

950

951

Lleres, D., J. James, S. Swift, D.G. Norman, and A.I. Lamond. 2009. Quantitative analysis of chromatin compaction in living cells using FLIM-FRET. J. Cell Biol. 187:481-496.

Maddox, P.S., N. Portier, A. Desai, and K. Oegema. 2006. Molecular analysis of mitotic chromosome condensation using a quantitative time-resolved fluorescence microscopy assay. Proc. Natl. Acad. Sci. U.S.A. 103:1509715102.

Markaki, Y., A. Christogianni, A.S. Politou, and S.D. Georgatos. 2009. Phosphorylation of histone H3 at Thr3 is part of a combinatorial pattern that marks and configures mitotic chromatin. J. Cell Sci. 122:2809-2819.

Martin, R.M., and M.C. Cardoso. 2010. Chromatin condensation modulates access and binding of nuclear proteins. FASEB J. 24:1066-1072.

Molnar, J., A. Poti, O. Pipek, M. Krzystanek, N. Kanu, C. Swanton, G.E. Tusnady, Z. Szallasi, I. Csabai, and D. Szuts. 2014. The Genome of the Chicken DT40 Bursal Lymphoma Cell Line. G3-Genes Genomes Genetics. 4:2231-2240.

Nagasaka, K., M.J. Hossain, M.J. Roberti, J. Ellenberg, and T. Hirota. 2016. Sister chromatid resolution is an intrinsic part of chromosome organization in prophase. Nature Cell Biology. 18:692-+.

Naumova, N., M. Imakaev, G. Fudenberg, Y. Zhan, B.R. Lajoie, L.A. Mirny, and J. Dekker. 2013. Organization of the mitotic chromosome. Science. 342:948953.

Nicklas, R.B., and C.A. Koch. 1969. Chromosome Micromanipulation III. Spindle fiber tension and the reorientation of mal-oriented chromosomes. J. Cell Biol. 43:40-50.

Nishihashi, A., T. Haraguchi, Y. Hiraoka, T. Ikemura, V. Regnier, H. Dodson, W.C. Earnshaw, and T. Fukagawa. 2002. CENP-I is essential for centromere function in vertebrate cells. Dev. Cell. 2:463-476.

Nishimura, K., T. Fukagawa, H. Takisawa, T. Kakimoto, and M. Kanemaki. 2009. An auxin-based degron system for the rapid depletion of proteins in nonplant cells. Nat. Methods. 6:917-922.

Nurse, P., and Y. Bissett. 1981. Gene required in G1 for commitment to cell cycle and in G2 for control of mitosis in fission yeast. Nature. 292:558-60.

Ohta, S., J.C. Bukowski-Wills, L. Sanchez-Pulido, L. Alves Fde, L. Wood, Z.A. Chen, M. Platani, L. Fischer, D.F. Hudson, C.P. Ponting, T. Fukagawa, W.C. Earnshaw, and J. Rappsilber. 2010. The protein composition of mitotic chromosomes determined using multiclassifier combinatorial proteomics. Cell. 142:810-821.

Oliveira, R.A., P.A. Coelho, and C.E. Sunkel. 2005. The condensin I subunit Barren/CAP-H is essential for the structural integrity of centromeric heterochromatin during mitosis. Mol. Cell. Biol. 25:8971-8984.

Ono, T., Y. Fang, D.L. Spector, and T. Hirano. 2004. Spatial and temporal regulation of Condensins I and II in mitotic chromosome assembly in human cells. Mol. Biol. Cell. 15:3296-308.

Ono, T., A. Losada, M. Hirano, M.P. Myers, A.F. Neuwald, and T. Hirano. 2003. Differential Contributions of Condensin I and Condensin II to Mitotic Chromosome Architecture in Vertebrate Cells. Cell. 115:109-121.

Paulson, J.R., and U.K. Laemmli. 1977. The structure of histone-depleted chromosomes. Cell. 12:817-828. 
958

959

960

961

962

963

964

965

966

967

968

969

970

971

972

973

974

975

976

977

978

979

980

981

982

983

984

985

986

987

988

989

990

991

992

993

994

995

996

997

998

999

Ribeiro, S.A., J.C. Gatlin, Y. Dong, A. Joglekar, L. Cameron, D.F. Hudson, C.J. Farr, B.F. McEwen, E.D. Salmon, W.C. Earnshaw, and P. Vagnarelli. 2009. Condensin regulates the stiffness of vertebrate centromeres. Mol. Biol. Cell. 20:2371-2380.

Saitoh, N., I. Goldberg, E. Wood, and W. Earnshaw, C. 1994. ScII: an abundant chromosome scaffold protein is a member of a family of putative ATPases with an unusual predicted tertiary structure. J. Cell Biol. 127:303-318.

Saka, Y., T. Sutani, Y. Yamashita, S. Saitoh, M. Takeuchi, Y. Nakaseko, and M. Yanagida. 1994. Fission yeast cut3 and cut14, members of the ubiquitous protein family, are required for chromosome condensation and segregation in mitosis. The EMBO J. 13:4938-4952.

Samejima, I., C. Spanos, L. Alves Fde, T. Hori, M. Perpelescu, J. Zou, J. Rappsilber, T. Fukagawa, and W.C. Earnshaw. 2015. Whole-proteome genetic analysis of dependencies in assembly of a vertebrate kinetochore. J Cell Biol. 211:1141-56.

Samejima, K., H. Ogawa, C.A. Cooke, D. Hudson, F. Macisaac, S.A. Ribeiro, P. Vagnarelli, S. Cardinale, A. Kerr, F. Lai, S. Ruchaud, Z. Yue, and W.C. Earnshaw. 2008. A promoter-hijack strategy for conditional shutdown of multiply spliced essential cell cycle genes. Proc. Natl. Acad. Sci. U.S.A. 105:2457-2462.

Samejima, K., I. Samejima, P. Vagnarelli, H. Ogawa, G. Vargiu, D.A. Kelly, F. de Lima Alves, A. Kerr, L.C. Green, D.F. Hudson, S. Ohta, C.A. Cooke, C.J. Farr, J. Rappsilber, and W.C. Earnshaw. 2012. Mitotic chromosomes are compacted laterally by KIF4 and condensin and axially by topoisomerase Ilalpha. J. Cell Biol. 199:755-770.

Samoshkin, A., A. Arnaoutov, L.E. Jansen, I. Ouspenski, L. Dye, T. Karpova, J. McNally, M. Dasso, D.W. Cleveland, and A. Strunnikov. 2009. Human condensin function is essential for centromeric chromatin assembly and proper sister kinetochore orientation. PLoS One. 4:e6831.

Saunders, W.S., and M.A. Hoyt. 1992. Kinesin-related proteins required for structural integrity of the mitotic spindle. Cell. 70:451-8.

Smith, E., N. Hegarat, C. Vesely, I. Roseboom, C. Larch, H. Streicher, K. Straatman, H. Flynn, M. Skehel, T. Hirota, R. Kuriyama, and H. Hochegger. 2011. Differential control of Eg5-dependent centrosome separation by Plk1 and Cdk1. EMBO J. 30:2233-45.

Somma, M.P., B. Fasulo, G. Siriaco, and G. Cenci. 2003. Chromosome condensation defects in barren RNA-interfered Drosophila cells. Genetics. 165:1607-11.

Sonoda, E., M.S. Sasaki, J.M. Buerstedde, O. Bezzubova, A. Shinohara, H. Ogawa, M. Takata, Y. Yamaguchi-Iwai, and S. Takeda. 1998. Rad51-deficient vertebrate cells accumulate chromosomal breaks prior to cell death. Embo J. 17:598-608.

Steffensen, S., P.A. Coelho, N. Cobbe, S. Vass, M. Costa, B. Hassan, S.N. Prokopenko, H. Hugo Bellen, M.M.S. Heck, and C.E. Sunkel. 2001. A role for Drosophila SMC4 in the resolution of sister chromatids in mitosis. Curr. Biol. 11:295307.

Sullivan, M., T. Higuchi, V.L. Katis, and F. Uhlmann. 2004. Cdc14 phosphatase induces rDNA condensation and resolves cohesin-independent cohesion during budding yeast anaphase. Cell. 117:471-482. 
1000 Sutani, T., T. Yuasa, T. Tomonaga, N. Dohmae, K. Takio, and M. Yanagida. 1999.

1001

1002

1003

1004

1005

1006

1007

1008

1009

1010

1011

1012

1013

1014

1015

1016

1017

1018

1019

1020

1021

1022

1023

1024

1025

1026

1027

1028

1029 Fission yeast condensin complex: essential roles of non-SMC subunits for condensation and Cdc2 phosphorylation of Cut3/SMC4. Genes Dev. 13:2271-2283.

Vagnarelli, P. 2012. Mitotic chromosome condensation in vertebrates. Experimental Cell Research. 318:1435-1441.

Vagnarelli, P., and W.C. Earnshaw. 2012. Repo-Man-PP1: a link between chromatin remodelling and nuclear envelope reassembly. Nucleus. 3:138142.

Vagnarelli, P., D.F. Hudson, S.A. Ribeiro, L. Trinkle-Mulcahy, J.M. Spence, F. Lai, C.J. Farr, A.I. Lamond, and W.C. Earnshaw. 2006. Condensin and Repo-ManPP1 co-operate in the regulation of chromosome architecture during mitosis. Nat. Cell Biol. 8:1133-1142.

Vassilev, L.T., C. Tovar, S. Chen, D. Knezevic, X. Zhao, H. Sun, D.C. Heimbrook, and L. Chen. 2006. Selective small-molecule inhibitor reveals critical mitotic functions of human CDK1. Proc Natl Acad Sci U S A. 103:10660-5.

West, J.B., Z.X. Fu, T.J. Deerinck, M.R. Mackey, J.T. Obayashi, and M.H. Ellisman. 2010. Structure-function studies of blood and air capillaries in chicken lung using 3D electron microscopy. Respiratory Physiology \& Neurobiology. 170:202-209.

Wood, L., D.G. Booth, G. Vargiu, S. Ohta, F. deLima Alves, K. Samejima, T. Fukagawa, J. Rappsilber, and W.C. Earnshaw. 2016. Auxin/AID versus conventional knockouts: distinguishing the roles of CENP-T/W in mitotic kinetochore assembly and stability. Open Biol. 6:150230.

Zhiteneva, A, J.J. Bonfiglio, A. Makarov, T. Colby, P. Vagnarelli, E.C. Schirmer, I. Matic and W.C. Earnshaw. 2017 . Mitotic post-translational modifications of histones promote chromatin compaction in vitro Open Biol. In press 
1030 FIGURE LEGENDS

1031 Figure 1. Rapid depletion of SMC2-mAID-GFP upon auxin treatment results in a

1032 severely defective mitotic chromosome formation. (A) Diagram introducing SMC2-

1033 AID-GFP cells. Auxin addition recruits the miniAID tag to the $\operatorname{SCF}^{\text {TIR1 }}$ complex,

1034 resulting in rapid degradation of SMC2-mAID-GFP via the proteasome pathway. (B)

1035 Growth curve of wild type cells and SMC2-AID-GFP cells treated with either ethanol

1036 (Et: solvent) or $125 \mu \mathrm{M}$ auxin (Au). Error bars show standard deviation. (C)

1037 Immunoblot analysis of asynchronous wild type and SMC2-AID-GFP cells. SMC2-AID-

1038 GFP cells were treated with $125 \mu \mathrm{M}$ auxin for $0-3 \mathrm{~h}$. SMC2 and SMC2-mAID-GFP

1039 were detected with anti-SMC2 antibody. $\alpha$-tubulin was a loading control. (D) Live cell

1040 imaging of a SMC2-AID-GFP cell by Zeiss Airy microscope. DNA was stained with SiR-

1041 DNA. SMC2-mAID-GFP concentrated along the axis of sister chromatids. Scale bar 1

$1042 \mu \mathrm{m}$. (E) SMC2-AID-GFP cells treated with ethanol (solvent) (i, ii) or auxin for $3 \mathrm{~h}$ (iii,

1043 iv) were fixed with formaldehyde and stained for $\alpha$-tubulin (green), and Histone 3

1044 phospho-Ser10 (red) and DNA (blue). The GFP signal was undetectable (iii, iv) and

1045 the shape of mitotic chromosomes (iii) was highly abnormal in SMC2-depleted cells.

1046 Scale bar $5 \mu \mathrm{m}$. (F) SMC2 ${ }^{\text {ON/OFF } / C E N P-H-G F P ~ c e l l s ~(V a g n a r e l l i ~ e t ~ a l ., ~ 2006) ~ c o n v e r t e d ~}$

1047 to $C D K 1^{\text {as }}$ were treated with doxycycline for 0 or $30 \mathrm{~h}$, stained in metaphase and

1048 anaphase for DNA (blue), CENP-H-GFP (green) and $\alpha$-tubulin (red). (G) Wild type/

1049 CDK1 ${ }^{\text {as }}$ cells ( 0 or $3 \mathrm{~h}$ auxin treatment), SMC2-AID-GFP/CDK1 ${ }^{\text {as }}$ cells ( 0 or $3 \mathrm{~h}$ auxin

1050 treatment) cells and SMC2 ${ }^{\mathrm{ON} / \mathrm{OFF}} / \mathrm{CDK} 1^{\text {as }}$ cells (0 or $34 \mathrm{~h}$ doxycycline treatment) were

1051 fixed with cold Methanol/Acetic acid and stained for DNA. More pictures in Figure1-

1052 figure supplement 1B. Scale bar $5 \mu \mathrm{m}$.

1053

1054 Figure 2. SMC2-depleted cells accumulate at prometaphase with a single

1055 chromosome mass. (A) Phosphorylation of Histone 3 Serine10 (red) was not affected

1056 by SMC2 depletion. DNA shown in blue and CENP-H-GFP in green. Interphase cells

1057 (i), prophase cells (ii) and prometaphase cells (iii). Control: SMC2 ${ }^{\mathrm{ON}} / \mathrm{CDK} 1^{\text {as }} / \mathrm{CENP}-\mathrm{H}-$

1058 GFP cells. SMC2-depleted: SMC2-AID-GFP/CDK1 ${ }^{\text {as }} /$ CENP-H-GFP cells. (B)

1059 Experimental timeline for auxin treatment. After 1NMPP1 washout, SMC2-

1060 AID/CDK1 ${ }^{\text {as }}$ cells were fixed with $4 \%$ formaldehyde and stained with corresponding

1061 antibodies. (C) Cell cycle and morphological analysis. > 200 cells were counted for 
1062 each time point for each treatment treated as in (B). Average of three independent

1063 experiments. Error bars represent standard deviation (SD). (D) Representative

1064 pictures of control cells and SMC2-depleted cells at different stages of mitosis. DNA

1065 (blue), CENP-T (red), $\alpha$-tubulin (green). Coloured box at the bottom right corners of

1066 merge panels correspond to the stages of mitosis in (C). SMC2-depleted mitotic cells

1067 have a single chromosome mass adjacent to a malformed mitotic spindle. Stretched

1068 chromatin fiber (arrow in i, right). Normal prometaphase-like chromosomes were

1069 occasionally observed within the auxin-treated cell population but all these cells

1070 were SMC2-mAID-GFP positive. (E) Stills from live-cell imaging of SMC2-AID-

$1071 \mathrm{GFP} / \mathrm{CDK} 1^{\text {as }}$ expressing Pericentrin/AKAP450 centrosomal targeting (PACT)-RFP

1072 treated with auxin $3-6 \mathrm{~h}$. DNA was stained with SiR-DNA. 3D image stacks were

1073 collected at $0.4-\mu \mathrm{m} z$ increments on a Zeiss Airy microscope every 5 mins. SMC2-AID-

$1074 \mathrm{GFP} / \mathrm{CDK} 1^{\text {as }}$ cells exited mitosis without apparent chromosome segregation.

1075 Chromosomes appeared to be decondensed and centrosome positioned close by in

1076 later time points (xii-xiv) Control (no auxin) is shown in Figure 2-figure supplement

1077 1D. Scale bars $5 \mu \mathrm{m}$.

1078

1079 Figure 3. Malformed and mis-positioned chromosomes and mitotic spindle in

1080 SMC2-depleted cells. (A) SMC2 ${ }^{\mathrm{ON}} / \mathrm{CDK} 1^{\text {as }} / \mathrm{CENP-H-GFP}$ cells and SMC2-AID-

1081 GFP/CDK1 ${ }^{\text {as }} /$ CENP-H-GFP (treated with auxin) were fixed with formaldehyde and

1082 stained for $\alpha$-tubulin (red) and DNA (blue). Images were acquired by structural

1083 illumination microscopy. (B) SMC2-AID-GFP/CDK1 ${ }^{\text {as }}$ expressing PACT-RFP was treated

1084 with 1NMPP1 for $4 \mathrm{~h}$ with or without auxin for $3 \mathrm{~h}$. These cells were fixed with

1085 formaldehyde $1 \mathrm{~h}$ after 1NMPP1 washout and stained for $\alpha$-tubulin (green), CENP-T

1086 (white), DNA (blue). Cells were 3D-rotated so that two PACT-RFP signals overlap (i,

1087 iii) or position in parallel (ii, iv). Note: Signals appeared to be fuzzier and stretched

1088 due to 3D-rotation. (C) SMC2-AID-GFP/CDK1 ${ }^{\text {as }}$ expressing PACT-RFP was treated as

1089 (B) and stained for DNA. Angles of chromosomes and centrosomes were calculated

1090 as described in Materials and Methods. Control $(n=22)$, SMC2-depleted $(n=26)$. P

1091 value was <0.0001 (two-tailed Mann-Whitney U Test). (D) Control and auxin-treated

1092 cells treated with nocodazole for $1 \mathrm{~h}$ and fixed with formaldehyde. DNA (blue),

1093 CENP-T (green), H3S10ph (red). (E) Experimental timeline for CENP-I depletion. (F, G) 
1094 SMC2-AID-GFP/CDK1 ${ }^{\text {as }}$ cells were transfected either with control siRNA oligo (F) or

1095 CENP-I oligo (G). Cells were further treated with ethanol or auxin. The chromosome

1096 mass became symmetrical in SMC2-depleted cells when kinetochore binding to

1097 microtubules was perturbed. CENP-T signals were enhanced in (i) in order to

1098 visualise the position of kinetochores. Non-enhanced images are also shown (ii). DNA

1099 (blue), CENP-T (red), $\alpha$-tubulin (green) Scale bars $5 \mu \mathrm{m}$.

1100

1101

Figure 4. Compromised kinetochore-microtubule attachments in SMC2-depleted

1102 cells. (A) Correlative light and transmission electron microscopy of control and SMC2

1103 depleted cells. Images show three progressive zooms of the white-boxed region, for

1104 TEM (i), light microscopy of DAPI (red) and CENP-H-GFP (green) (ii) and a correlative

1105 overlay of both the physical and optical sections (iii). Arrow in zoom 2 points to a

1106 clearly defined trilaminar structure. Scale bars A - 3 $\mu \mathrm{m}, 1 \mu \mathrm{m}, 200 \mathrm{~nm}$ for overview,

1107 zoom 1 and zoom 2 respectively. (B) Enlargement of kinetochores shown in Figure 4.

1108 Microtubules (yellow lines) and kinetochore (green) were overlaid on the TEM

1109 pictures, and show microtubule attachment. Scale bar $200 \mathrm{~nm}$. (C)

$1110 \mathrm{SMC2}^{\mathrm{ON}} / \mathrm{CDK}^{1}$ as $/$ CENP-H-GFP cells and SMC2-AID-GFP/CDK1 $1^{\text {as }} / \mathrm{CENP-H-GFP}$ (treated

1111 with auxin) were treated with 1NMPP1 for $4 \mathrm{~h}$ and fixed with formaldehyde $1 \mathrm{~h}$ after

1112 1NMPP1 washout. Strong MAD2 staining on the centromeres inside of the SMC2-

1113 depleted chromosome mass (yellow arrowheads) is not observed on centromeres

1114 outside of the chromosome mass (white arrows). DNA (blue), MAD2 (red), CENP-H-

1115 GFP (green). Scale bar $5 \mu \mathrm{m}$.

1117 Figure 5. 3D-EM shows that condensin is required for chromosome organization,

1118 but not for chromatin compaction. WT and condensin depleted cells were prepared

1119 for 3D SBF-SEM analysis. (A) A control cell with all chromosomes modeled (indigo) is

1120 shown at different angles, with and without the EM orthoslice ( $i-i v)$. Centrioles

1121 (green) and Pericentriolar material (orange) are also shown. Chromosomes were

1122 separated into individual units (multi-coloured) ( $v$-vi). (B) A condensin depleted cell

1123 with all chromosomes modeled (red), shown at different angles, with and without

1124 the EM orthoslice ( $\mathrm{i}-\mathrm{iii}, \mathrm{v}$-vii). Separated chromosomes traversing an EM orthoslice

1125 (iv and viii). (C) SMC2-depleted chromosomes were separated into discrete units. (D) 
1126 2D scatter plot of surface area versus volume for individual chromosomes in A

1127 (control, green) and B (condensin depleted, magenta). Scale bar $5 \mu \mathrm{m}$.

1128

1129

Figure 6. SMC2 is required to maintain the structure of mitotic chromosomes. (A)

1130 The intrinsic mitotic chromosome structure (IMCS) assay. Cells were blocked in

1131 mitosis with nocodazole. Removal of cations in hypotonic buffer induced the

1132 unfolding of chromatin to the level of $10 \mathrm{~nm}$ fibres. Addition of $\mathrm{Mg}^{2+}$ triggered the

1133 shrinking and refolding of chromatin. The shape of chromosomes after two cycles of

1134 unfolding and folding was classified as reformed (green), partially reformed (yellow)

1135 or disorganised (magenta). (B) Representative images of DAPI-stained mitotic

1136 chromosomes at the end of the assay. Scale bar $5 \mu \mathrm{m}$. (C) Quantification of

1137 chromosomes at the end of the assay. The graph shows the mean and SD of three

1138 independent experiments with > 50 cells/each treatment counted. (D) Experimental

1139 procedure for determining the role of condensin in establishing and maintaining

1140 mitotic chromosome structure. Cultures were treated with 1NMPP1 for $13 \mathrm{~h}$. After

1141 1NMPP1 washout, cultures were treated with nocodazole for $4 \mathrm{~h}$ to hold the cells in

1142 mitosis $(T=0.5-4.5 \mathrm{~h}$ ). The mitotic index reached approximately $80 \%$ after $1 \mathrm{~h}$ of

1143 nocodazole addition and did not change until nocodazole washout at $4.5 \mathrm{~h}$. Cells

1144 were fixed with formaldehyde at indicated time points and stained with DAPI before

$1145>100$ cells/each treatment's time point were counted. Mean and SD of three

1146 independent experiments are shown at F and Figure 6-figure supplement 2B-D. (E)

1147 Cells were fixed with formaldehyde/PBS without hypotonic treatment at the

1148 indicated time points and stained with DAPI. (F) The amount of SMC2 remaining was

1149 measured by immunoblot analysis and normalized to the level of $\alpha$-tubulin. (G, H)

1150 Cells were treated with $75 \mathrm{mM} \mathrm{KCl}$ at $37^{\circ} \mathrm{C}$ for 5 min prior to ice-cold

1151 methanol/acetic acid fixation and DAPI staining.

1152

1153 Figure 7. Cells partially depleted of SMC2 show chromosome mis-segregation. (A)

1154 The amount of SMC2 remaining in asynchronous culture upon $4 \mathrm{~h}$ auxin treatment

1155 was measured by immunoblot analysis and normalized to the level of $\alpha$-tubulin.

1156 Mean and SD of three independent experiments are shown. (B) Representative

1157 images of SMC2-AID-GFP cells at prometaphase upon $4 \mathrm{~h}$ auxin treatment with 
1158 various concentrations of auxin. SMC2-mAID-GFP (green). Scale bar $5 \mu \mathrm{m}$. (C) Effect

1159 of various auxin concentrations on mitotic progression of SMC2-AID-GFP cells.

1160 Experimental procedure as in Figure 2B. Cells were fixed every $30 \mathrm{~min}$ after 1NMPP1

1161 washout. Auxin was omitted from the media used for the wash and release except in

1162 the case of cells treated with $250 \mu \mathrm{M}$ auxin. Mean and SD of three independent

1163 experiments. $>200$ cells were counted for each time point of individual treatments.

1164

1165 Figure 8. Differing amounts of condensin are required for chromosome assembly

1166 and for segregation. (A) Experimental protocol. (B) 45 min after 1NMPP1 washout,

1167 cells were subjected to the IMCS assay. Representative images are shown. > 100

1168 cells/each treatment were counted. Mean and SD of three independent

1169 experiments. (C) 90 min after 1NMPP1 washout, cells were fixed and stained with

1170 anti $\alpha$-tubulin antibody and DAPI. Representative images are shown. $>20$ anaphase

1171 or telophase cells/each treatment were counted. Cultures treated with $125 \mu \mathrm{M}$ auxin

1172 did not show anaphase/telophase cells, so cells in early cytokinesis (iv) were

1173 analysed. Mean and SD of three independent experiments. (D) Overlay of key

1174 phenotypes from the experiments of $(B, C)$ plotted against the residual levels of

1175 SMC2-mAID-GFP measured by immunoblotting and normalized to the level of $\alpha$ -

1176 tubulin in cells treated with the indicated concentration of auxin.

1178 Figure 9 Effects of lowering condensin levels on chromosome structure and

1179 segregation (A) SMC2-AID-GFP/CDK1 ${ }^{\text {as }}$ cells were treated with 1 NMPP1 for $13 \mathrm{~h}$ and

1180 with the indicated amounts of auxin for the final $3 \mathrm{~h}$. Auxin was omitted from media

1181 used for the wash and release except for cells treated with $125 \mu \mathrm{M}$ auxin. $30 \mathrm{~min}$

1182 after 1NMPP1 washout, cells were collected, rinsed with PBS and fixed with cold

1183 methanol/acetic acid. DNA (blue), SMC2 (red), and Top $2 \alpha$ (green). Scale bar $5 \mu \mathrm{m}$. (B)

1184 Diagram summarizing the changes in protein distribution seen in (A). (C) Summary of

1185 the effects of differing condensin levels on mitotic DT40 cells. DNA (blue),

1186 centromeres (green), mitotic spindle (red). 
1189 Figure supplement:

1190 Figure 1-figure supplement 1. Characterisation of SMC2-AID-GFP cell line. (A)

1191 Asynchronous wild type/CDK1 ${ }^{\text {as }}$ cells and SMC2-AID-GFP/ CDK1 ${ }^{\text {as }}$ cells were fixed

1192 with formaldehyde and stained with DNA (blue), $\alpha$-tubulin (green) and H3S10ph

1193 (red). 500 cells were counted for each sample. Mean and SD from three independent

1194 experiments are shown. (B) Cells were treated as Figure 1G. Scale bar $5 \mu \mathrm{m}$. (C) Flow

1195 cytometry analysis of GFP positive cells treated with auxin for 0-3 $\mathrm{h}$.

1196

1197 Figure 2-figure supplement 1. Mitotic cells depleted of SMC2. (A) INCENP (red)

1198 localises on chromosomes in early mitosis in SMC2-AID-GFP/CDK1 ${ }^{\text {as }} /$ CENP-H-GFP

1199 cells both treated or not treated with auxin. Control (no auxin) and SMC2-depleted

1200 (auxin $3 \mathrm{~h}$ ). (B) Asynchronous wild type/CDK1 ${ }^{\text {as }}$ cells were treated with 1 NMPP1 for 0

1201 or 30 min and stained for DNA (blue) and H3S10ph (red). (C) Analysis of DNA content

1202 of wild type/CDK $1^{\text {as }}$ cells (wt) and SMC2-AID-GFP/CDK1 ${ }^{\text {as }}$ cells using flow cytometry.

1203 Cells from asynchronously growing culture (i), $4 \mathrm{~h}$ block and release from 1NMPP1

1204 (ii), or $4 \mathrm{~h}$ block and release from 1NMPP1 with auxin (iii) were analysed as described

1205 in Materials and Methods. Positions corresponding cells with 2N DNA (blue arrow) or

1206 4N DNA (red arrow) are shown. (D) Stills from live-cell imaging of SMC2-AID-

1207 GFP/CDK1 ${ }^{\text {as }}$ expressing Pericentrin/AKAP450 centrosomal targeting (PACT)-RFP

1208 (control). DNA was stained with SiR-DNA. 3D image stacks were collected at 0.4- $\mu \mathrm{m} \mathrm{z}$

1209 increments on a Zeiss Airy microscope every 5 mins. SMC2-AID-GFP/CDK1 ${ }^{\text {as }}$ cells

1210 exited mitosis ( $v-x$ ). Stills of an SMC2-depleted cell (auxin) is shown in Figure 2E. (E)

1211 SMC2-AID-GFP cells treated with auxin for 2 days. Scale bars $5 \mu \mathrm{m}$.

1212

1213 Figure 3-movie supplement.

$12143 \mathrm{D}$ reconstruction of control (1) and SMC2-depleted cells (2) shown in Figure 3A

1215 taken by 3D-SIM microscopy. DNA (blue), CENP-H-GFP (green), $\alpha$-tubulin (red).

1216

1217 Figure 4-figure supplement 1. SMC2-depleted cells exit mitosis with highly uneven

1218 chromosome segregation after Mad2 RNAi (A) INCENP concentrates on central

1219 spindle in cells exiting mitosis. Separate tubulin image is shown. Panels show (i) no

1220 auxin, control siRNA; (ii) auxin, control siRNA; (iii) no auxin, Mad2 siRNA; (iv) auxin, 
1221 Mad2 siRNA. DNA (blue), CENP-H-GFP + SMC2mAID-GFP (green), INCENP (red), $\alpha-$

1222 tubulin (grey). Scale bar $5 \mu \mathrm{m}$. (B) Cyclin B2 levels fall in cells exiting mitosis.

1223 Separate tubulin image is shown. Panels show (i) no auxin, control siRNA; (ii) auxin,

1224 control siRNA; (iii) no auxin, Mad2 siRNA; (iv) auxin, Mad2 siRNA. DNA (blue), CENP-

1225 H-GFP + SMC2-mAID-GFP (green), Cyclin B2 (red), $\alpha$-tubulin (grey). Scale bar $5 \mu \mathrm{m}$.

1226 (C) Immunoblot analysis showing MAD2 depletion by specific siRNA. (D)

1227 Even/uneven chromosome segregation was scored for $>50$ anaphase or telophase

1228 cells per sample. Mean and SD from three independent experiments are shown. (E)

1229 Quantification of live cell imaging. Cells were transiently transfected with Histone

1230 H2B-RFP.

1231

1232 Figure 6-figure supplement 1. ICMS assay after nocodazole block (A) SMC2-AID-GFP

1233 cells were treated with nocodazole for $12 \mathrm{~h}$. Auxin was added at the indicated time

1234 points. (B) Depletion of SMC2 was confirmed by immunoblot analysis. (C)

1235 Quantification of SMC2 relative to $\alpha$-tubulin based on (B). Mean and SD from three

1236 independent experiments are shown.

1237

1238 Figure 6-figure supplement 2. Mitotic exit of cells depleted of SMC2. (A)

1239 Experimental protocol. (B) The relative amount of SMC2 was measured by

1240 immunoblot analysis using anti-SMC2 and anti $\alpha$-tubulin antibodies. (C) The relative

1241 amount of CyclinB2 was measured by immunoblot analysis using anti-Cyclin B2 and

1242 anti $\alpha$-tubulin antibodies. (D) Cells were fixed with $4 \%$ formaldehyde at the time

1243 points indicated and stained by DAPI before counting. > 100 cells/sample were

1244 counted. For B-D, mean and SD from three independent experiments are shown. (E)

1245 Cells were fixed with formaldehyde in PBS without hypotonic treatment at the

1246 indicated time points and stained with anti-tubulin (red), Cyclin B2 antibodies (grey)

1247 and DAPI (blue). CENP-H-GFP + SMC2-mAID-GFP are green. Scale bar $5 \mu \mathrm{m}$. 


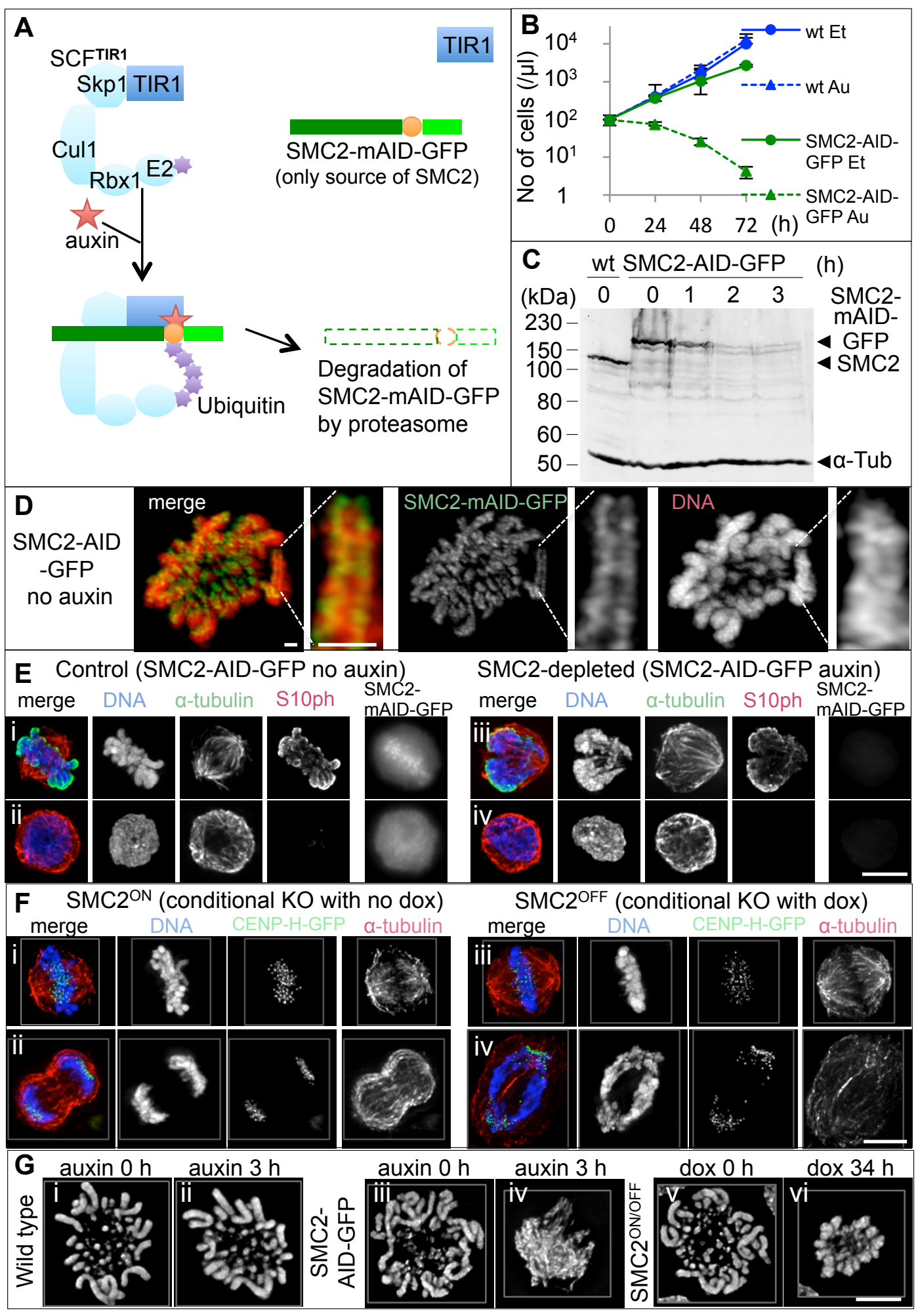



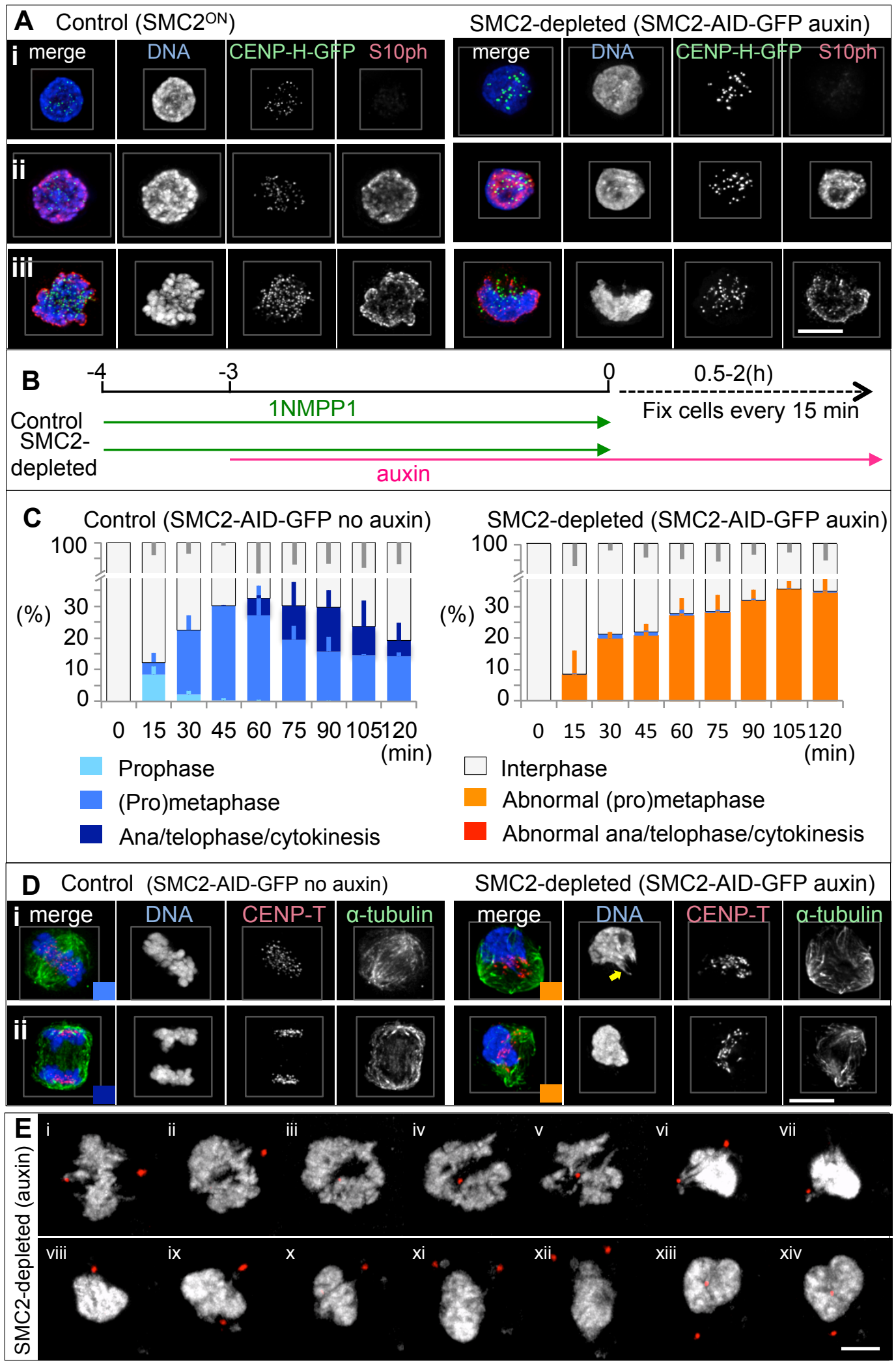

Samejima et al. Figure 2 


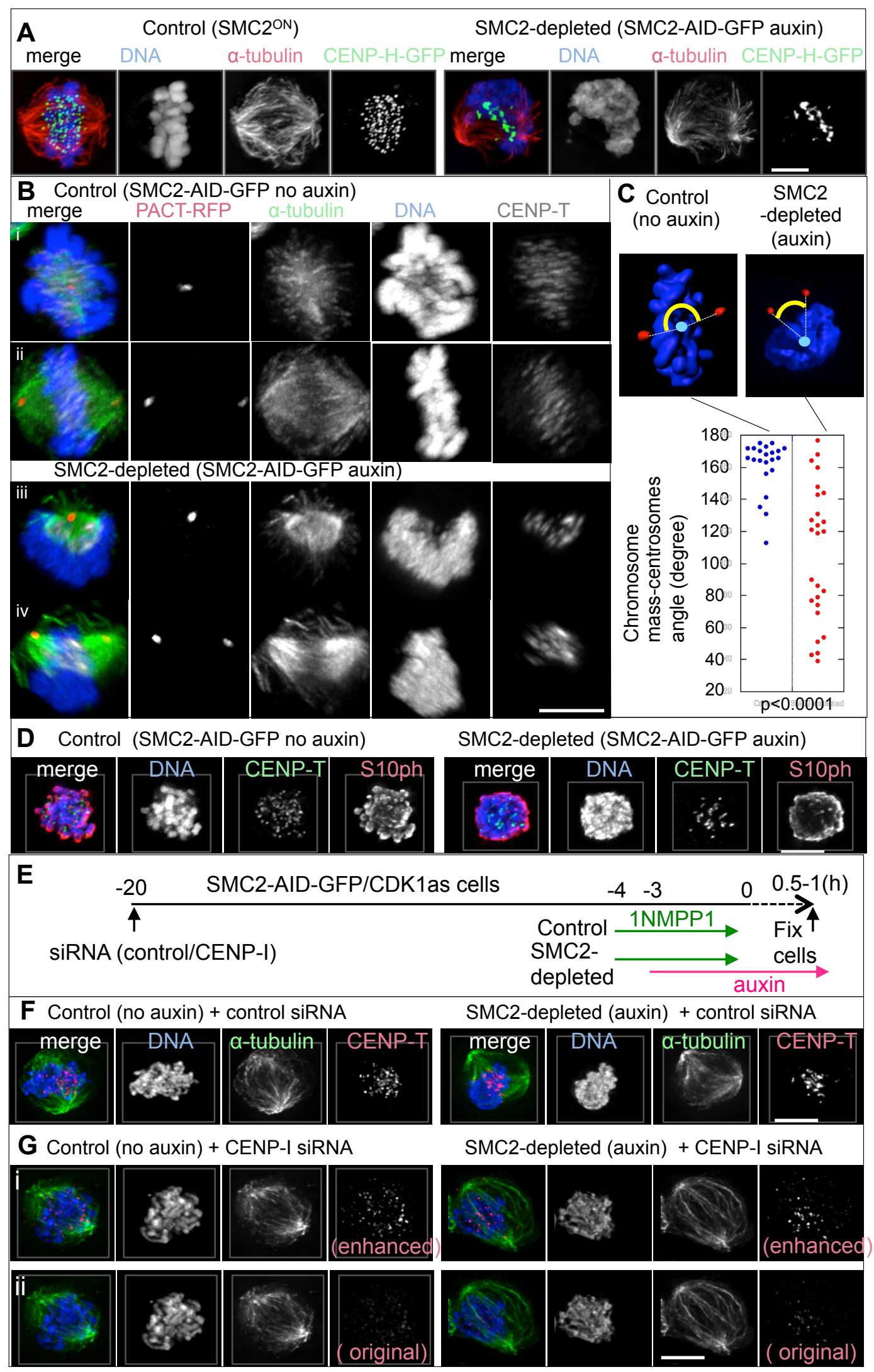

Samejima et al. Figure 3 

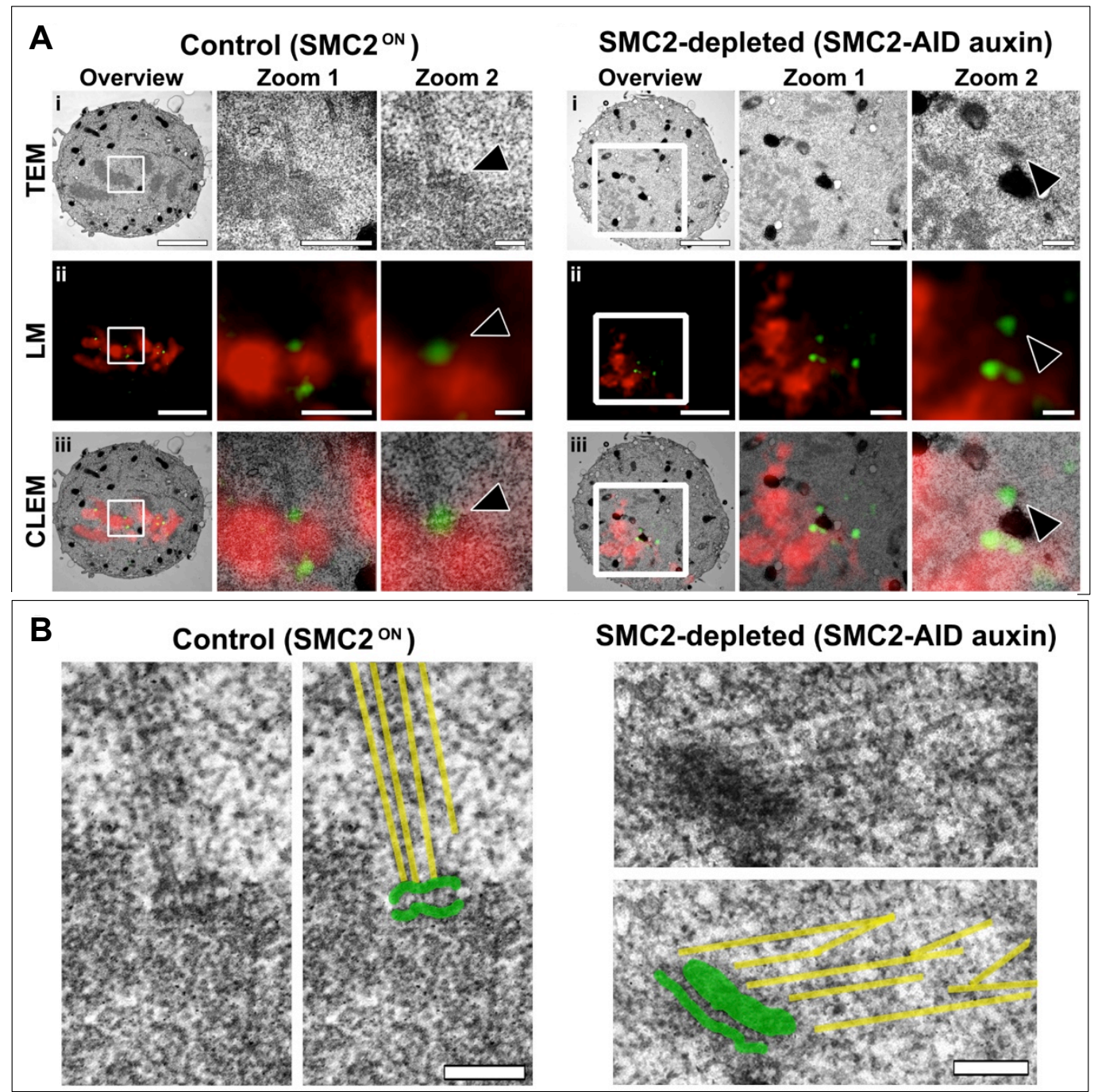

C Control (SMC2 $\left.{ }^{\mathrm{ON}}\right)$

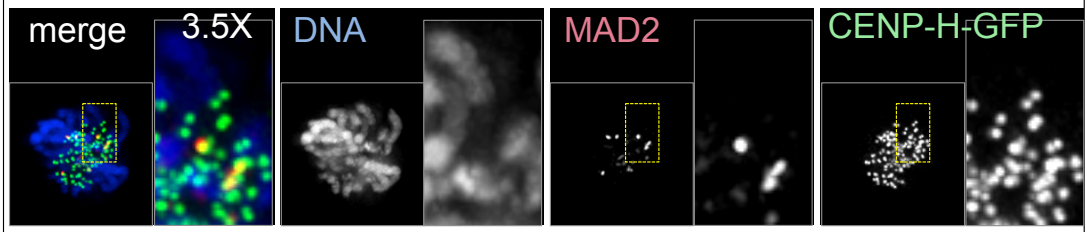

SMC2-depleted (SMC2-AID-GFP, auxin )
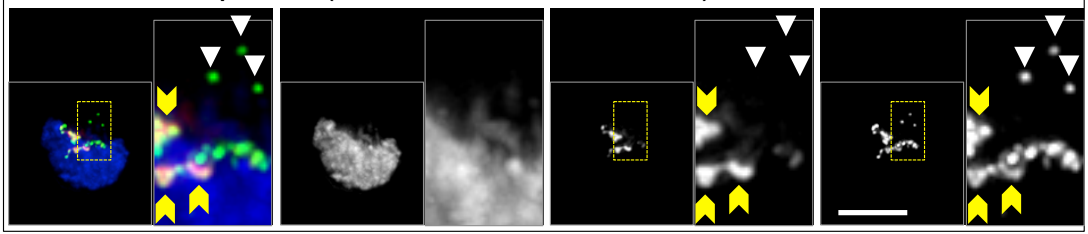

Samejima et al. Figure 4 

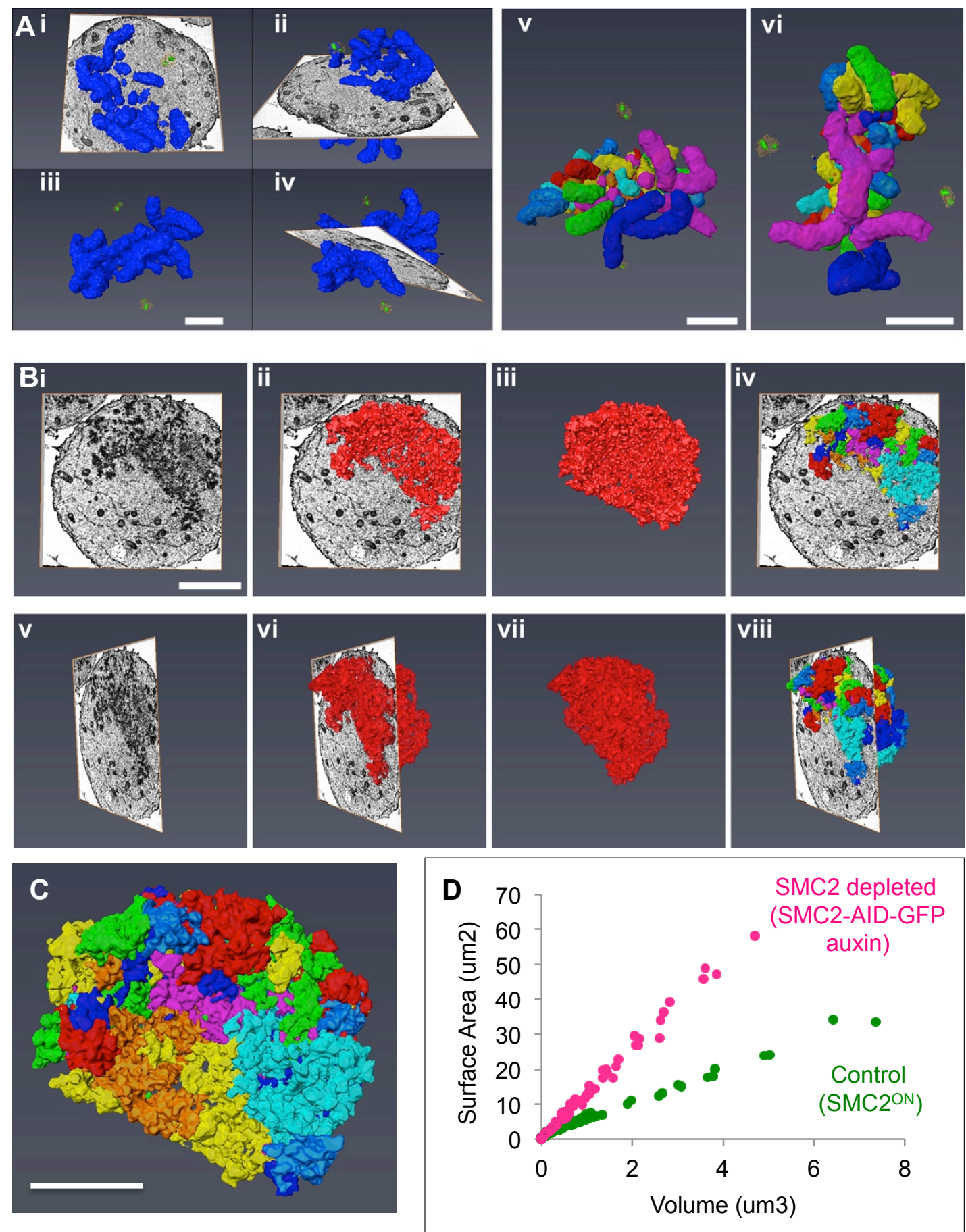

Samejima et al. Figure 5 


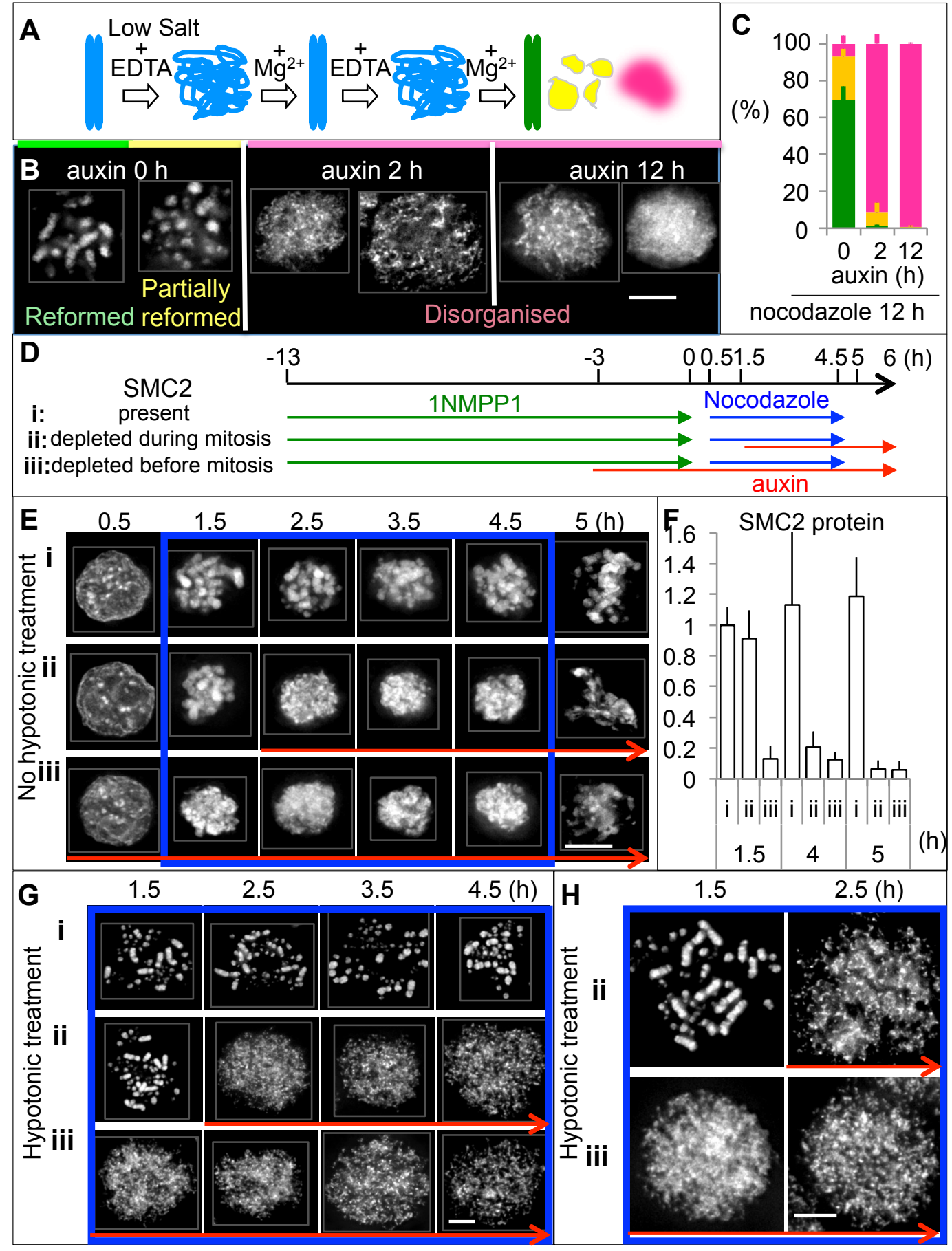

Samejima et al. Figure 6 


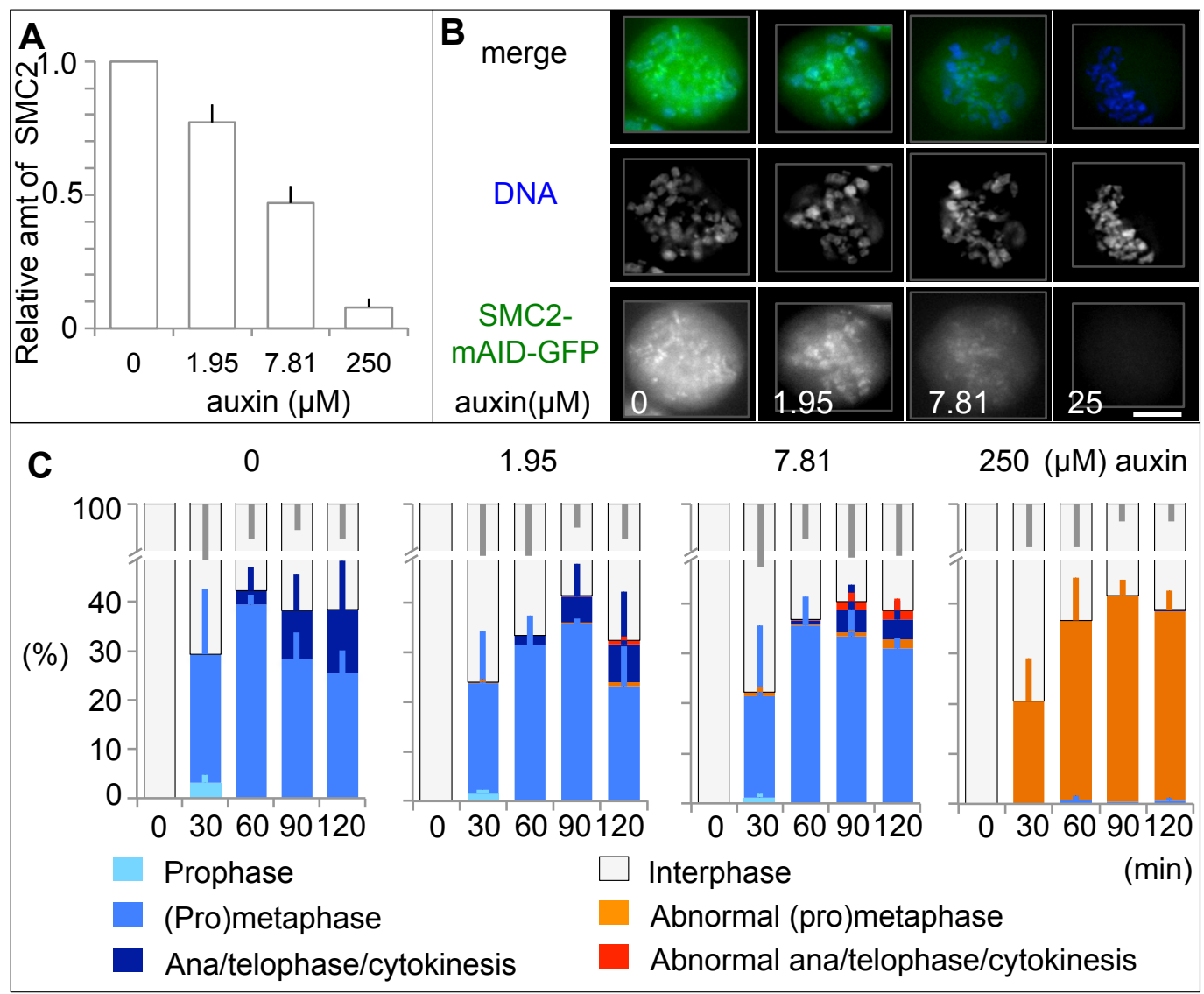

Samejima et al. Figure 7 

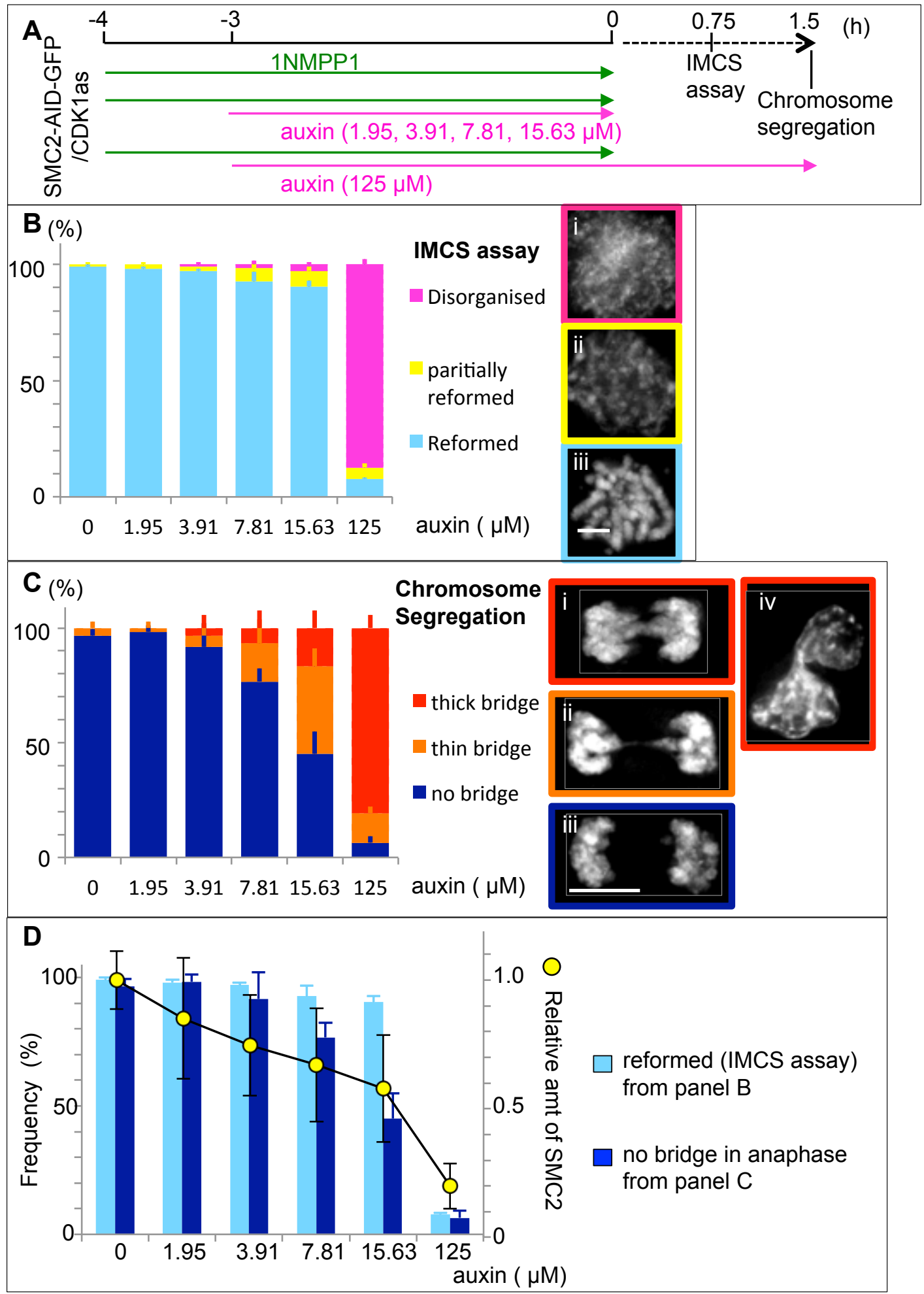


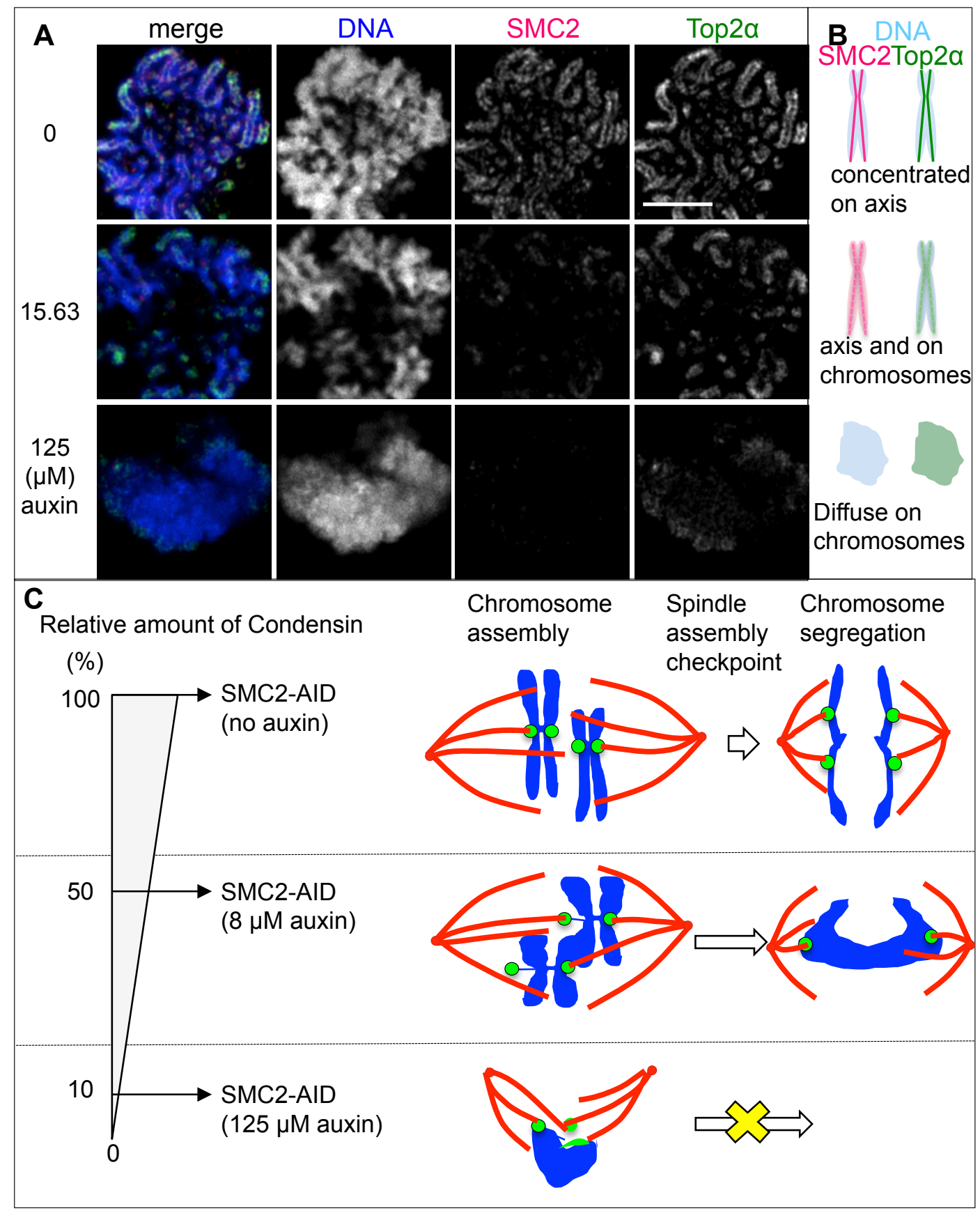

Samejima et al. Figure 9 


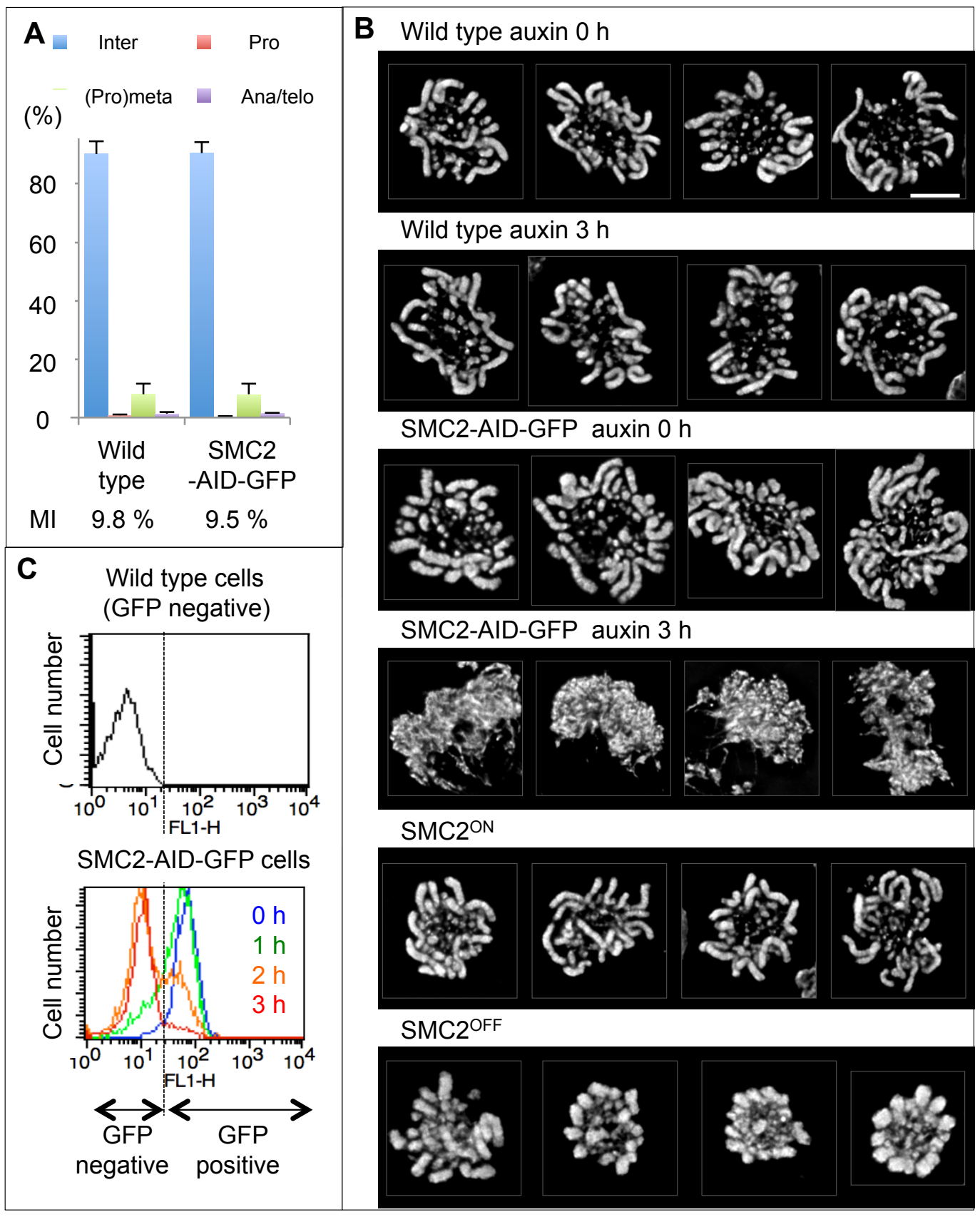

Samejima et al. Figure 1-figure supplement 1 


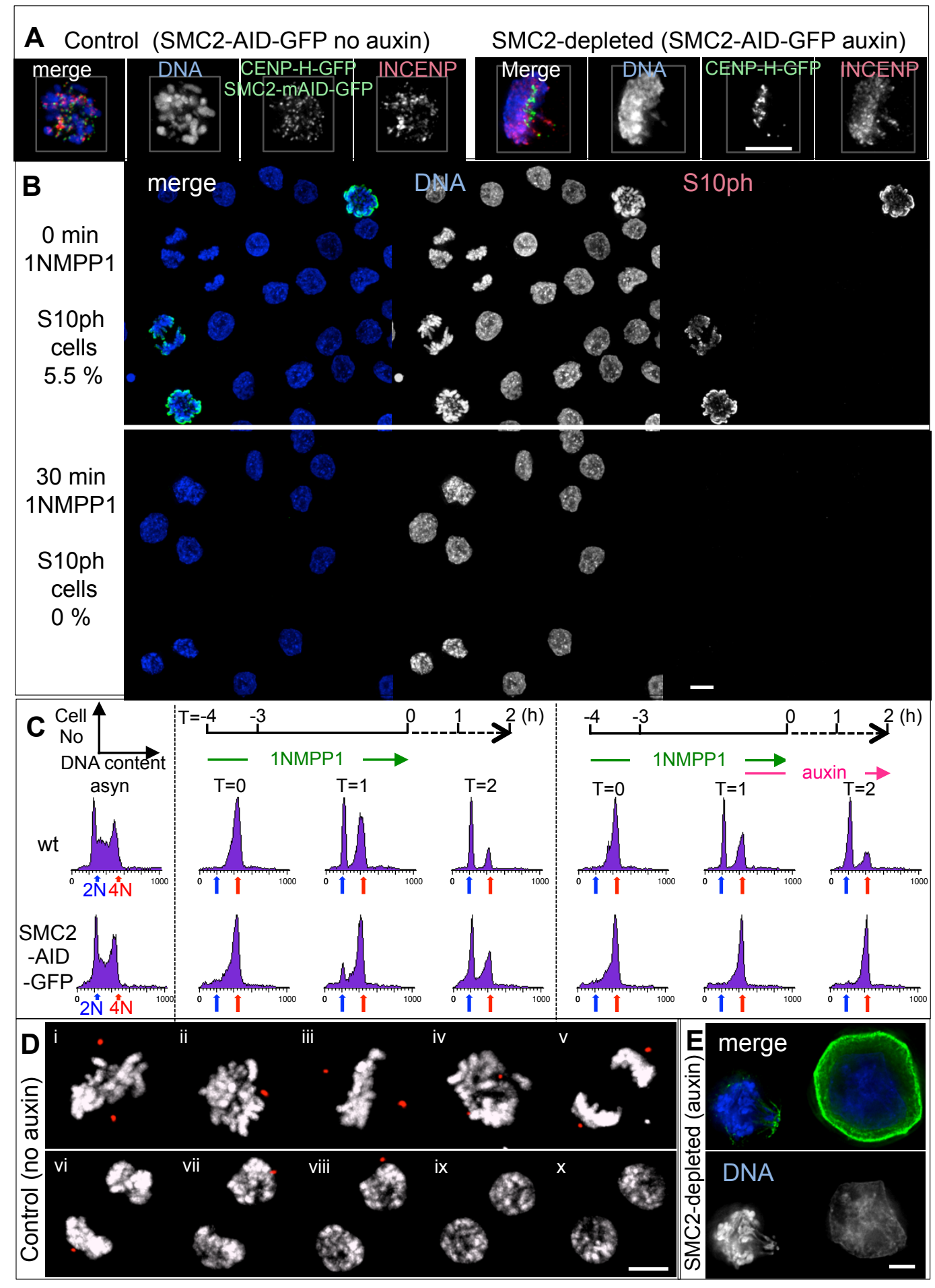

Samejima et al. Figure 2-figure supplement 1 


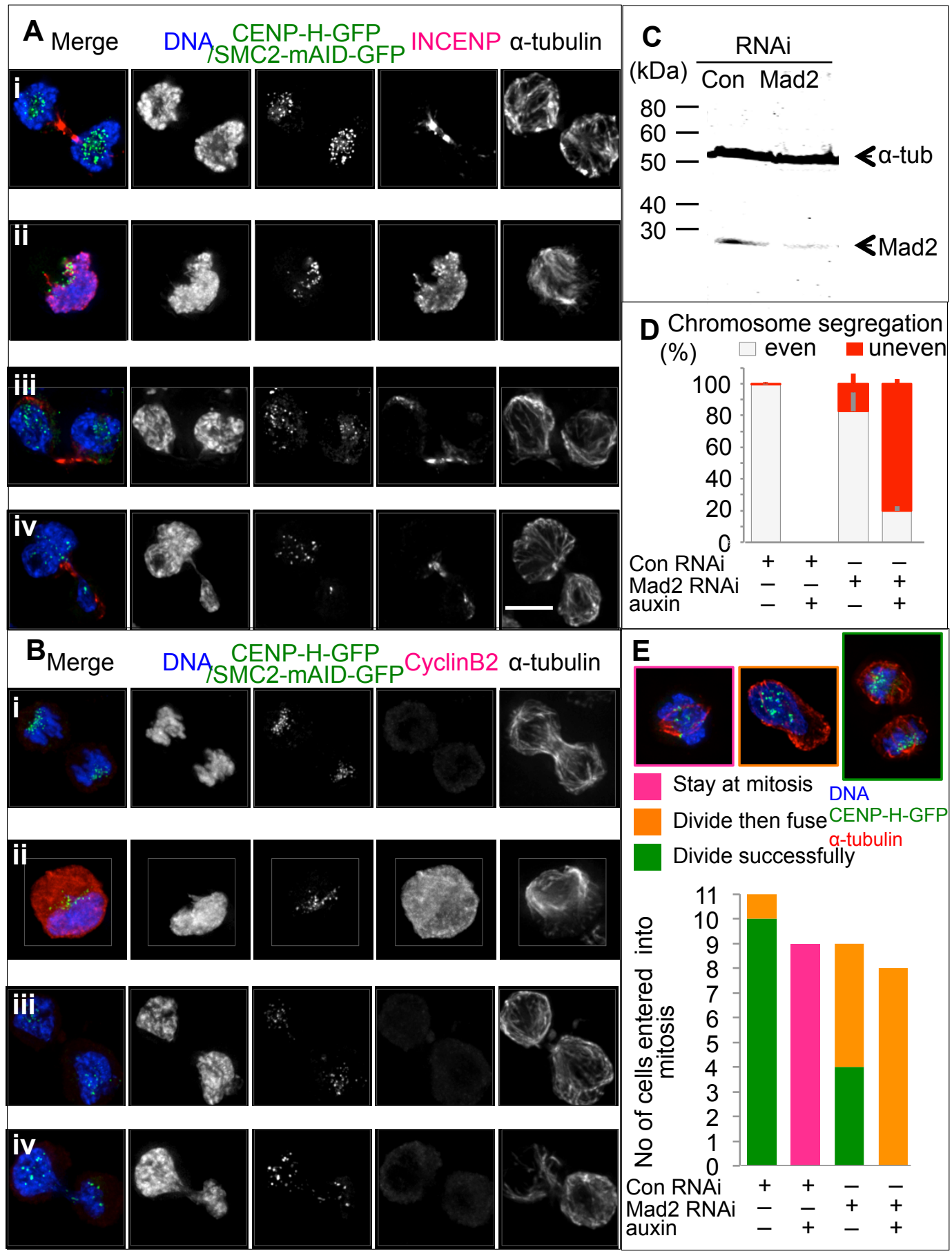

Samejima et al. Figure 4-figure supplement 1 


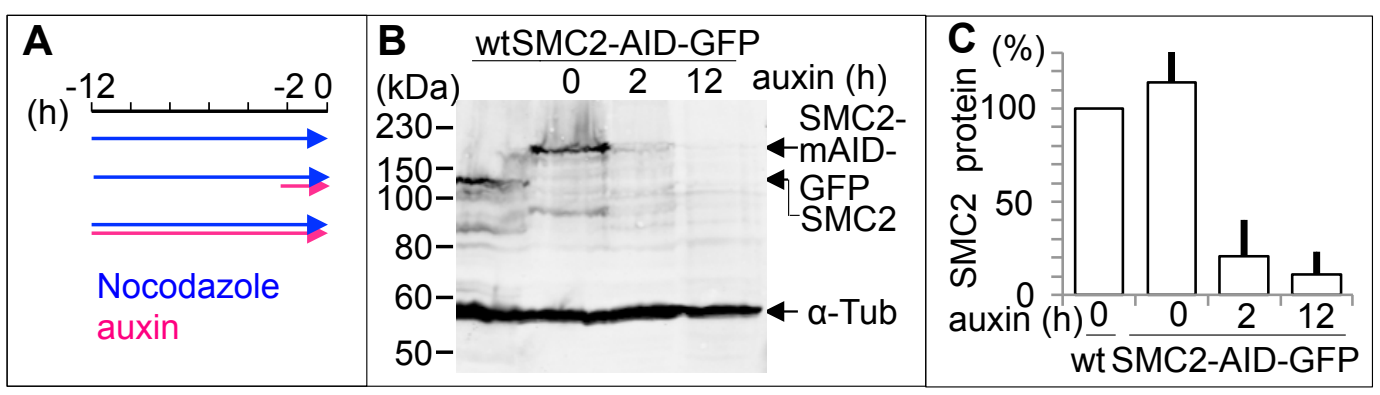

Samejima et al. Figure 6-figure supplement 1 


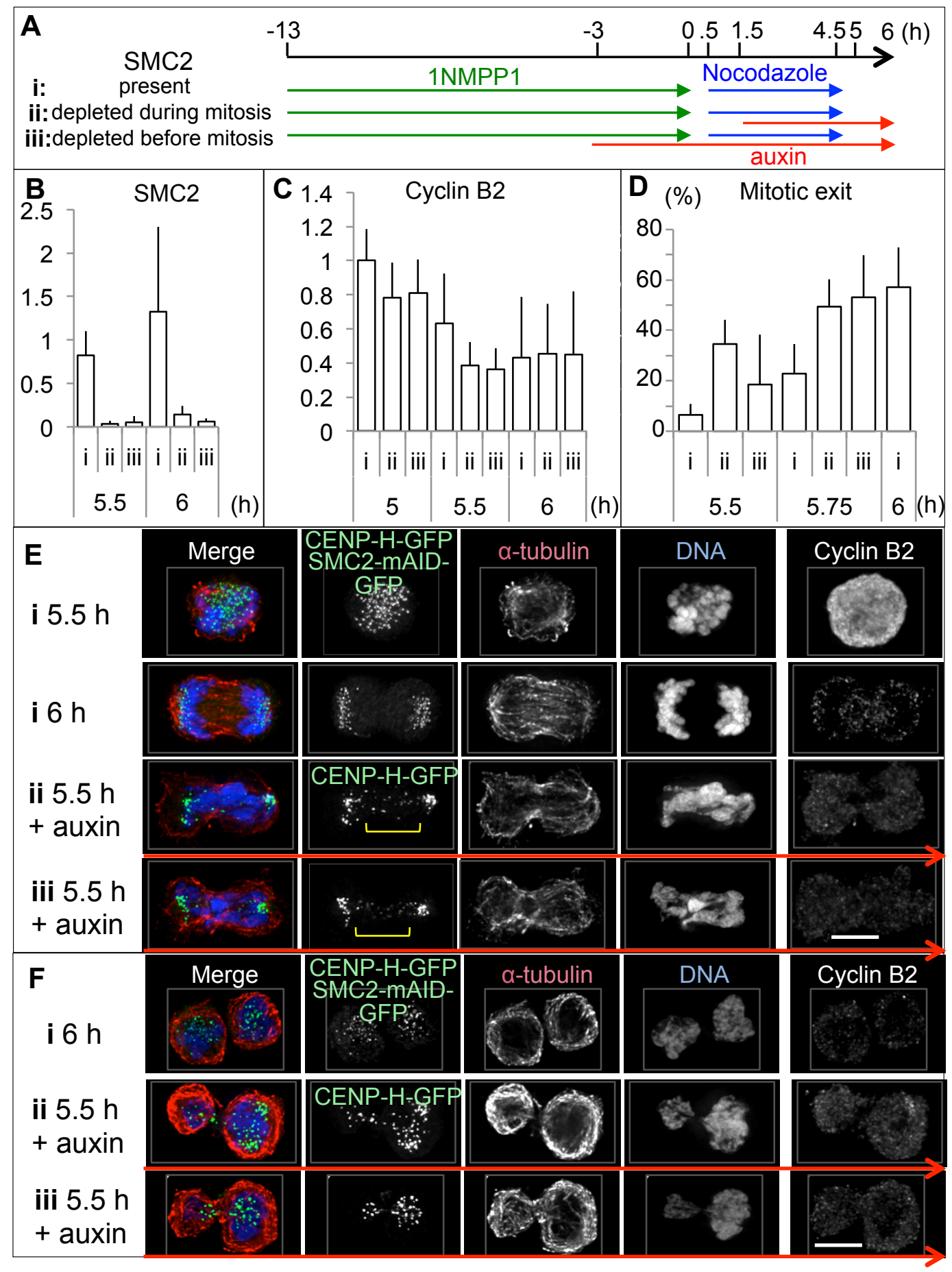

Samejima et al. Figure 6-figure supplement 2 